\title{
Scientific Data Management Integrated Software Infrastructure Center (SDM/ISIC):
}

\section{Scientific Process Automation (SPA) \\ FINAL REPORT}

\author{
to the US Department of Energy SciDAC Program \\ Grant \# DE-FC02-01ER25486
}

Bertram Ludäscher* Ilkay Altintas ${ }^{\dagger}$

$08 / 15 / 2001-08 / 14 / 2007$

\section{Contents}

1 Executive Summary 1

2 Background 3

3 Scientific Workflows 3

3.1 Example Workflows . . . . . . . . . . . . . . . . . . . . . . . . 3

3.1 Promoter Identification . . . . . . . . . . . . . . . . . . . . . . 3

3.1 .2 Mineral Classification . . . . . . . . . . . . . . . . . . . . 5

3.1.3 Job Scheduling . . . . . . . . . . . . . . . . . . . . . . . . 5

3.2 Requirements and Desiderata . . . . . . . . . . . . . . . . . . . . . 6

3.3 Differences to Business Workflows . . . . . . . . . . . . . . . . . . . . . . 8

4 SPA Technology Development 9

4.1 Web Service Extensions . . . . . . . . . . . . . . . . . . . . . . . 9

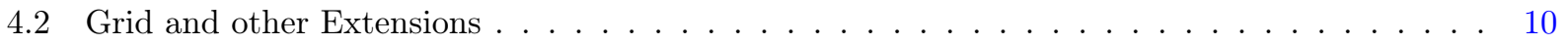

4.3 Actor-Oriented Modeling . . . . . . . . . . . . . . . . . . . . 11

5 Research Issues 13

5.1 Higher-Order Constructs . . . . . . . . . . . . . . . . . . . . . . . . . 13

5.2 Third Party Transfers . . . . . . . . . . . . . . . . . . . . . . . . . . . 14

5.3 Other Research Issues . . . . . . . . . . . . . . . . . . . . . . . . . . 15

5.4 Related Work . . . . . . . . . . . . . . . . . . . . . . . 16

6 Brief History of the KEPLER Collaboration 16

7 Excerpts from the Quarterly Reports 18

8 Selected Publications 20

9 Concluding Remarks 20

*Dept. of Computer Science, UC Davis, ludaesch@ucdavis.edu (until 2004: San Diego Supercomputer Center, UCSD)

†San Diego Supercomputer Center, UCSD; altintas@sdsc.edu 


\section{Executive Summary}

The KePLeR project ${ }^{1}$ has made a significant impact and established a sustained leadership in the field of scientific workflows. The open source KEPLER project started in 2003 when members of the SciDACSDM Center/SPA Team, sponsored by this DOEfunded project (DE-FC02-01ER25486) and members of the SEEK project (NSF/ITR awards DBI-0225674 and DBI-0533368) decided to collaborate and jointly develop a scientific workflow system based on the open source Ptolemy II system (Ludäscher was a co-PI on both projects). In the first project phase, between 2001 and 2003, the SDSC team (Altintas, Ludäscher) worked closely with a domain scientist (Matt Coleman, LLNL) and created early versions of a Promoter Identification Workflow (PIW) [15, 62]. Towards the end of that period, the open source PTOLEMY II system was adopted by SciDAC-SDM and SEEK as the basis for a general scientific workflow system and problem-solving environment to design and execute scientific workflows, giving rise to Kepler.

Once started through SciDAC-SDM and SEEK, the KEPLER leadership team was able to attract further support from funding agencies including from DOE (as part of SciDAC-2), NSF, NIH, and the Gordon and Betty Moore Foundation. Important advances to KEPLER were made by the SciDAC/SDMSPA team during the report period (2001-2007), in particular by the SDSC team (and after Ludäscher's move) the UC Davis team, including (i) research and development of the workflow infrastructure, (ii) library (actor) development, and (iii) development of concrete scientific workflows, in collaboration with scientists. Years later, the KePLER system was listed as one of the prominent "Big Data" research outcomes of DOE in the White House Big Data Factsheet, announced as part of the White House Big Data press release $[21] .^{2}$

In addition to the core technology development, research in scientific workflow management was conducted and led to a deeper understanding of fundamental technical challenges in scientific workflow

\footnotetext{
${ }^{1}$ http://kepler-project.org

${ }^{2}$ Quoting from [22]: "The Office of Advanced Scientific Computing Research (ASCR) provides leadership to the data management, visualization and data analytics communities, including digital preservation and community access. Programs within the suite include widely used data management technologies such as the Kepler scientific workflow system; Storage Resource Management standard; a variety of data storage management technologies, such as BeSTman, the Bulk Data Mover and the Adaptable IO System (ADIOS); FastBit data indexing technology (used by Yahoo!); and two major scientific visualization tools, ParaView and VisIt."
}

design and execution. Research results have been documented in a number of publications and presented at national and international meetings and conferences including several at the Supercomputing Conference (SC), the International Conference on Scientific and Statistical Database Management (SSDBM), the e-Science Workflow Services Workshop [4], the e-Science Grid Environments Workshop [6], the Virtual Observatory Service Composition Workshop [7], the e-Science LINK-Up Workshop on Workflow Interoperability and Semantic Extensions [8], and the Global Grid Forum (GGF10) Scientific Workflow Workshop [9]. The success of the KEPLER collaboration for scientific workflow development is also documented by the 6th Biennial Ptolemy Miniconference that was featuring a special track on the KEPLER project [43] (KEPLER members also contributed to Ptolemy miniconferences in subsequent years). Theoretical results with applications in workflow design and composition have appeared in the Intl. Workshop on Data Integration in the Life Sciences [26], and database theory conferences [56, 32], among many others. ${ }^{3}$ In particular, the publications [49] and [13], led by Ludäscher and Altintas, respectively, are among the most cited papers on scientific workflows and provenance, according to Google Scholar.

Early Development Highlights. Initial development efforts of our team were focused on developing a number of generic workflow components, called actors, e.g., to support rapid workflow prototyping based on web services (web service actor, web service harvester); to integrate legacy applications via command line and ssh actors; to support user interaction via a BrowserUI actor; to support data-intensive workflows via SRB (Storage Resource Broker) actors; to support computeintensive workflows via a generic "Grid workflow" framework [16]; and to support data transformations via XSLT actors.

\section{Outreach and Growth of the KEPLER/SPA Col-} laboration. In collaboration with other KEPLER participants, a number of further components and workflows were created, e.g., a cheminformatics workflow to control high-end computing workflows [19, 20], several ecoinformatics and geoinformatics workflows, and workflows dealing with real-time sensor data. Dr. Ludäscher, then co-PI of the NSF/ITR projects GEON, SEEK, and ROADNet, had actively recruited these new communities to contribute to KEPLER. With his move to the Department of Computer

\footnotetext{
${ }^{3}$ See scholar.google.com/scholar?q=DE-FC02-01ER25486 for an online list.
} 
Science at UC Davis, KePLER members also joined from there, e.g., via the graduate seminar ECS-289F: Topics in Scientific Data Management and a new course ECS-166: Scientific Data Management for nonCS majors (Fall 2005). In both courses, students are trained by Dr. Ludäscher to use and develop new KEPLER/SPA workflows. Meanwhile, at SDSC, Ilkay Altintas has very actively recruited further groups and communities leading to additional members of the collaboration. Moreover, under guidance from Reagan Moore and Arcot Rajasekar from SDSC, Storage Resource Broker (SRB) components have been added to KEPLER in the early project phases.

Report Organization. Section 2 provides a highlevel introduction, motivating the need for scientific workflows. In Section 3 we introduce scientific workflows by means of several real-world examples from different domains. We use those examples to illustrate some of the characteristic features and requirements of scientific workflows, and compare the latter with business workflows. These sections are based on a preprint of Ludäscher et al. [49]. In Section 4 we describe some of the generic technology we have developed, in particular web service extensions (Section 4.1) and grid-related extensions (Section 4.2). We also introduce the notion of actor-oriented modeling, which is inherited from Ptolemy II. Section 5 presents some ongoing research issues. In Section 6 we discuss some details of the KEPLER collaboration of which SPA has been a founding member. A more detailed list of accomplishments is given in Section 7, derived from the quarterly reports. Section 8 highlights a few of the recent publications and presentations. Some concluding remarks are given in Section 9 . 
"The diversity of the phenomena of nature is so great, and the treasures hidden in the heavens so rich, precisely in order that the human mind shall never be lacking in fresh nourishment."

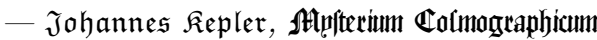

\section{Background}

Information technology is revolutionizing the way many sciences are conducted, as witnessed by new techniques, results, and discoveries from quickly evolving, multi-disciplinary fields such as bioinformatics, biomedical informatics, cheminformatics, ecoinformatics, geoinformatics, etc. To further advance this new data- and information-driven science through advanced IT infrastructure, large investments are made, e.g., in the UK e-Science programme, or in the US through the NSF Cyberinfrastructure initiative and other initiatives from NIH (BIRN: Biomedical Informatics Research Network) and DOE (SciDAC: Scientific Discovery through Advanced Computing, GTL: Genomes to Life), just to mention a few. While many efforts focus on the underlying middleware infrastructure, known as "the Grid", scientists are ultimately interested in tools that bring the power of distributed databases and other computational Grid resources to the desktop, and allow them to conveniently put together and run their own scientific workflows. By these we mean process networks that are typically used as "data analysis pipelines" or for comparing observed and predicted data, and that can include a wide range of components, e.g., for querying databases, for data transformation and data mining steps, for execution of simulation codes on high performance computers, etc. Ideally, the scientist should be able to plug-in almost any scientific data resource and computational service into a scientific workflow, inspect and visualize data on the fly as it is computed, make parameter changes when necessary and re-run only the affected "downstream" components, and capture sufficient metadata in the final products such that the runs of a scientific workflow, when considered as (computational) experiments themselves, help explain the results and make them reproducible by the computational scientist and others. Thus, a scientific workflow system becomes a scientific problemsolving environment, tuned to an increasingly distributed and service-oriented Grid infrastructure.

However, before this grand vision can become reality, a number of significant challenges have to be addressed. For example, current Grid software is still too complex to use for the average scientist, and fast changing versions and evolving standards require that these details be hidden from the user by the scientific workflow system. Web services seem to provide a simple basis for loosely coupled, distributed systems, but core web service standards such as WSDL [81] only provide simple solutions to simple problems, ${ }^{4}$ while harder problems such as web service orchestration, 3rd party transfer (from one service directly to another, circumventing the transfer back to a workflow control engine), and transactional semantics of service-based workflows, remain the subject of emerging or future web service standards. The complexity of the underlying technical issues and the resulting (sometimes overly) complex standards make it less likely that those will be as widely adopted as the core standards such as XML and WSDL.

Another set of challenges arises from the inherent complexity of scientific data itself. For example, how can we capture more of the semantics of scientific data (beyond simple metadata meant for human consumption) and thus inform the system which data sets might be suitable input for a specific analytical pipeline? Similarly, how can we define when it is even potentially meaningful at the conceptual level to compose two independently designed web services, or when an analysis pipeline might be included as a subworkflow in another scientific workflow? Knowledge representation techniques, including formal ontologies, and corresponding Semantic Web standards such as the Web Ontology Language [61] seem promising directions. However, as is the case for Grid middleware, the goal is to hide the underlying complexity as much as possible from the user of a scientific workflow system.

\section{Scientific Workflows}

In the following we first introduce scientific workflows by means of several examples taken from different projects and implemented using the Ptolemy IIbased KePler system [40]. We then discuss typical features of scientific workflows and from this derive general requirements and desiderata for scientific workflow systems. We take a closer look at underlying technical issues and challenges in Section 4.

\subsection{Example Workflows}

\subsubsection{Promoter Identification}

Figure 1 shows a high-level, conceptual view of a typical scientific knowledge discovery workflow that links genomic biology techniques such as microarrays with bioinformatics tools such as BLAST to identify and

\footnotetext{
${ }^{4}$ E.g. WSDL mainly provides an XML notation for function signatures, i.e., the types of inputs and outputs of web services.
} 


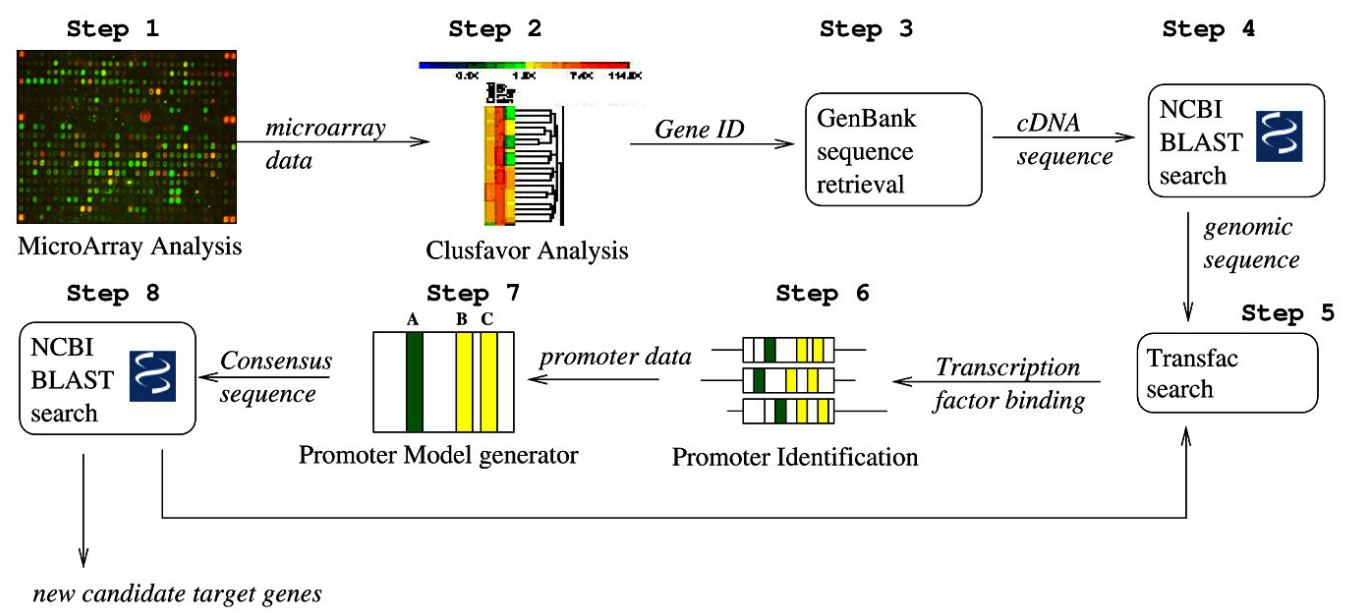

Figure 1: Conceptual ("napkin drawing") view of the Promoter Identification Workflow (PIW) [15]

characterize eukaryotic promoters ${ }^{5}$ - we call this the Promoter Identification Workflow or PIW (see also [79, 15, 62]: Starting from microarray data, cluster analysis algorithms are used to identify genes that share similar patterns of gene expression profiles that are then predicted to be co-regulated as part of an interactive biochemical pathway. Given the gene-ids, gene sequences are retrieved from a remote database (e.g., GenBank) and fed to a tool (e.g., BLAST) that finds similar sequences. In subsequent steps, transcription factor binding sites and promoters are identified to create a promoter model that can be iteratively refined.

While Figure 1 leaves many details open, some features of scientific workflows can already be identified: There are a number of existing databases (such as GenBank) and computational tools (such as Clusfavor and BLAST) that need to be combined in certain ways to create the desired workflow. In the past, accessing remote resources often meant implementing a wrapper that mimics a human entering the input of interest, submitting an HTML form, and "screen-scraping" the result from the returned page [45]. Today, more and more tools and databases become accessible via web services, greatly simplifying this task. Another trend is web portals such as NCBI [58] that integrate many tools and databases and sometimes provide the scientist with a "workbench" environment.

Figure 2 depicts snapshots of an early implementation of PIW in KEPLER. KEPLER is an extension of the Ptolemy il system [64] for scientific workflows. The topmost window includes a loop whose body is expanded below and which performs several steps on

\footnotetext{
${ }^{5} \mathrm{~A}$ promoter is a subsequence of a chromosome that sits close to a gene and regulates its activity.
}

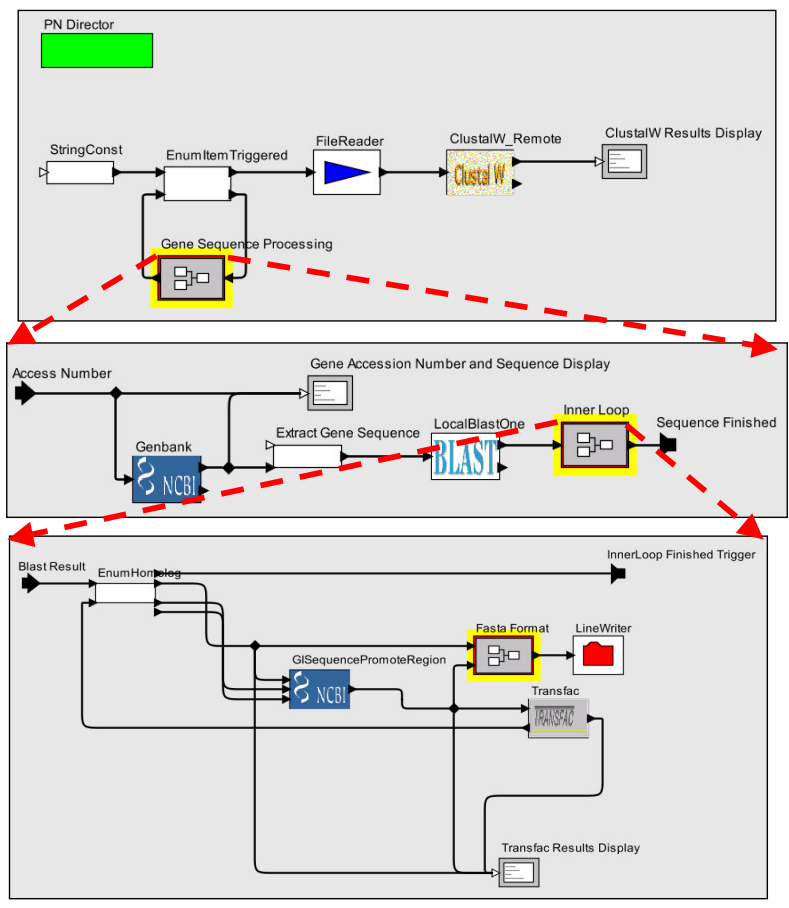

Figure 2: PIW implemented in KePler [15]. Composite actors (subworkflows) expanded below.

each of the given gene-ids: First, an NCBI web service is used to access GenBank data. Subsequently a BLAST step is performed to identify similar sequences to the one retrieved from GenBank. Then a second inner loop is executed (bottom window) for a transcription factor binding site analysis. Using Ptolemy II terminology, we call the individual steps actors, since they act as independent components which communicate with each other only through the channels indicated in the figure. The overall execution of the workflow is orchestrated by a director (the 
green box in Figure 2; see Section 4.3 for details).

This early PIW implementation in KePLER [15] illustrates a number of features: Actual "wiring" of a scientific workflow can be much more complicated than the conceptual view (Figure 1) suggests. A mechanism for collapsing details of a subworkflow into an abstract component (called composite actor in Ptolemy iI) is essential to tame complexity: The windows in Figure 2 have well-defined input and output ports and thus correspond to (sub)-workflows that can be collapsed into a more abstract, composite actor as indicated. Nevertheless, the resulting workflow is fairly complex and we will need to introduce additional mechanisms to simplify the design in particular of loops (see Section 5.1).

\subsubsection{Mineral Classification}

The second example, from a geoinformatics domain, illustrates the use of a scientific workflow system for automation of an otherwise manual procedure, or alternatively, for reengineering an existing custom tool in a more generic and extensible environment. The upper left window in Figure 3 shows the top-level workflow: Some samples are selected from a database holding experimentally determined mineral compositions of igneous rocks. This data, together with a set of classification diagrams are fed into a ClASSIFIER subworkflow (bottom left). The manual process of classifying samples involves determining the position of the sample values in a series of diagrams such as the one shown on the right in Figure 3: if the location of a sample point in a non-terminal diagram of order $n$ has been determined (e.g., diorite gabbro anorthosite, bottom right), the corresponding diagram of order $n+1$ is consulted and the point located therein. This process is iterated until the terminal level of diagrams is reached (here shown in the upper right: the classification result is anorthosite).

This traditionally manual process has been automated in commercial custom tools, or here in the KEPLER workflow shown in Figure 3. As above, workflows are shown in graphical form using PTOLEMY II's Vergil user interface [27]. Note that in Vergil, workflows can be annotated with user comments. Subworkflows (e.g., bottom-left) become visible by rightclicking on a composite actor (such as ClASSIFIER, upper-left) and selecting "Look Inside" from the resulting pop-up menu. Vergil also features simple VCRlike control buttons to play, pause, resume, and stop workflow execution (red icons in the top-left toolbar; e.g., right-triangle for play).

KEPLER specific features of this workflow include: A searchable library of actors and data sources (Actor and Data tabs close to the upper-left) with numerous reusable KEPLER actors. For example, the Browser actor (used in the bottom-right of the Classifier subworkflow) launches the user's default browser and can be used as a powerful generic input/output device in any workflow. In this example, the classification diagrams are generated on the client side as interactive SVG displays in the browser (windows on the right in Figure 3). Moving the mouse over the diagram highlights the specific region and displays the rock name classification(s) for that particular region. The BROwSER actor has proven to be very useful in many other workflows as well, e.g., as a device to display results of a previous step, and as a selection tool that passes user choices (made via HTML forms, check-boxes, etc.) to subsequent workflow steps.

\subsubsection{Job Scheduling}

The final example workflow, depicted in Figure 4, is from a cheminformatics domain and involves running thousands of jobs of the GAMESS quantum chemical code [68] under the control of the Nimrod/G Grid distribution tool [10]. This is an example of a workflow employing high-performance computing (HPC) resources in a coordinated manner to achieve a computationally hard task, in this case a variant of a hybrid quantum mechanics/molecular mechanics (QM/MM) technique; see [35] and [73] for details. Interestingly, the workflow in Figure 4 is rather domain-neutral and illustrates some features typical of many highperformance computational experiments:

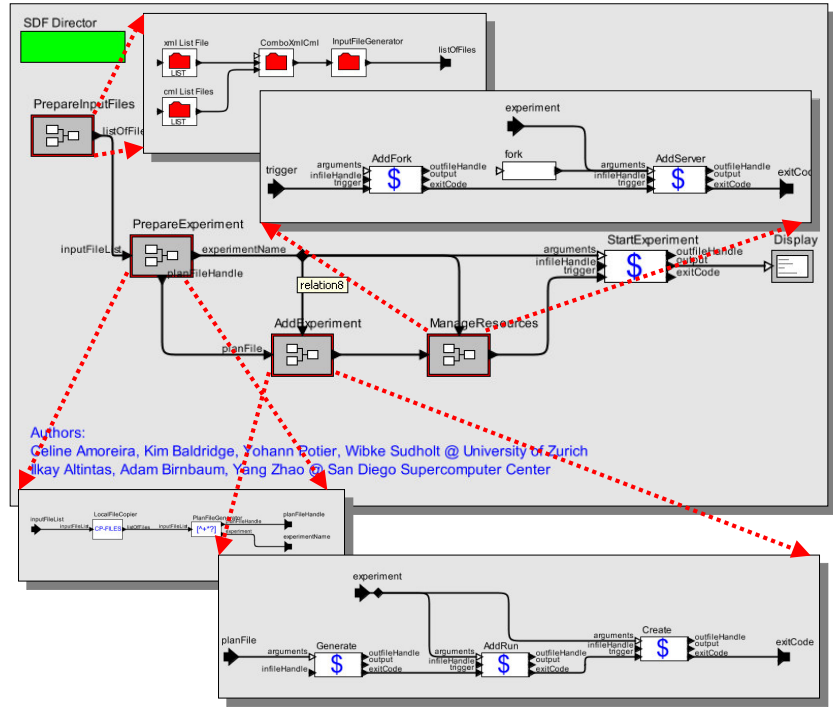

Figure 4: Workflow for scheduling HPC jobs.

The main window shows four composite actors, corresponding to the four depicted subworkflows. The 


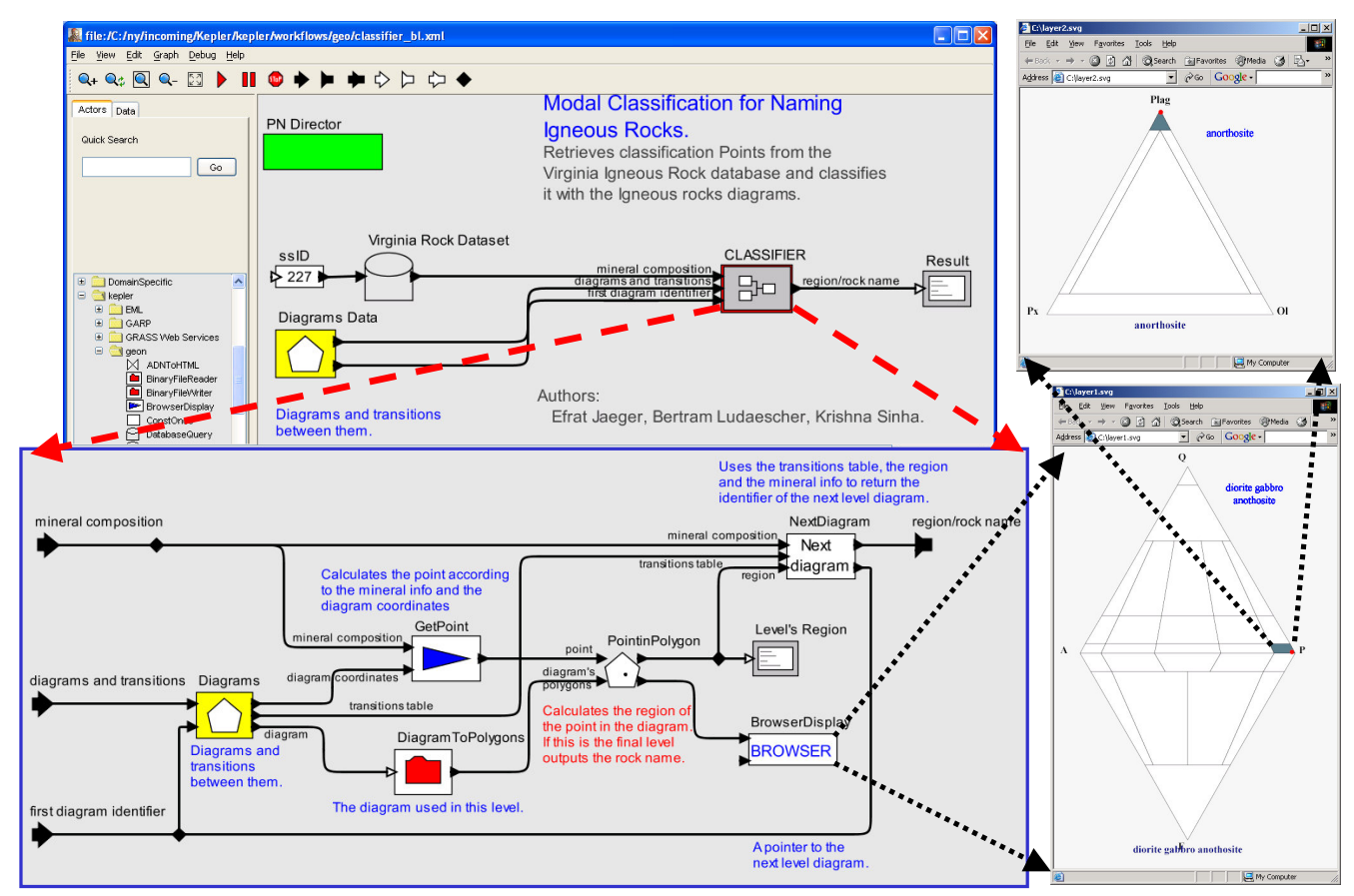

Figure 3: Mineral Classification workflow (left) and generated interactive result displays (right).

first one, PrepareInputs creates a list of input files for the subsequent jobs. These files are then used to create a plan file for Nimrod/G in the PREPAREEXPERIMENT step. The ADDEXPERIMENT subworkflow takes a plan file and generates experiment run files using several CommandLine actors. The latter is shown with a "\$" icon (to indicate a command shell), and has proven to be a very useful rapid-prototyping tool: Existing local applications can be made part of a workflow simply by providing a suitable command line expression and the corresponding command line arguments. The ManageRESOURCES subworkflow can create new processes (via ADDFORK) to run jobs and subsequently add experiments as new server processes.

This example workflow also highlights the possibility of incremental design and development: At the time of writing, not all components of the overall workflow are operational. Nevertheless, due to the clearly defined input/output interfaces of all subworkflows (a feature inherited from PTOLEMY II), each of them can be designed, implemented, and tested separately. Moreover, the current version of the workflow relies heavily on invoking external applications via the CommandLine actor. Some of these applications might be "promoted" to custom actors with native Java implementations in the future. Such changes are encapsulated by the containing subworkflow and thus do not require changes of other parts of the workflow.

\subsection{Requirements and Desiderata}

In this section we summarize a number of common requirements and desiderata of scientific workflows, as exhibited by the examples above or by other workflows we encountered in various application-oriented research projects including in addition to SDM/SPA also GEON, SEEK and several others [36, 70, 69, 23, 67].

\section{R1: Seamless access to resources and services:} This is a very common requirement (e.g., see the example workflows in Section 3.1), and web services provide a first, simple mechanism for remote service execution and remote database access $^{6}$ via service calls. However, as mentioned before, web services are a simple solution to a simple problem. Harder problems, e.g., web service orchestration, and 3rd party transfer are not solved by "vanilla" web services alone.

R2: Service composition \& reuse and workflow design: Since web services emerge as the basic building blocks for distributed Grid applications and workflows, the problem of service composition, i.e., how to compose simple services to perform complex tasks, has become

\footnotetext{
${ }^{6}$ We do not elaborate on the important challenges of data integration [71]; see, e.g., [37] for a survey of query rewriting techniques, and [57] and [51,25] for related issues of query capabilities and semantics, respectively.
} 
a hot research topic [38]. Among the different approaches are those that view service composition as an AI planning problem [24], a query planning problem [50, 52], or a general design and programming problem. A related issue is how to design components so that they are easily reusable and not geared to only the specific applications that may have driven their original development. As we will see, service composition and reuse are addressed by employing an actor-oriented approach at the design level (Section 4.3), but also require flexible means for data-transformations at the "plumbing" level (Section 4.2).

R3: Scalability: Some workflows involve large volumes of data and/or require high-end computational resources, e.g., running a large number of parallel jobs on a cluster computer (such as workflow in Section 3.1.3). To support such data-intensive and compute-intensive workflows, suitable interfaces to Grid middleware components (sometimes called Compute-Grid and DataGrid, respectively) are necessary.

R4: Detached execution: Long running workflows require an execution mode that allows the workflow control engine to run in the background on a remote server, without necessarily staying connected to a user's client application that has started and is controlling workflow execution (such as the Vergil GUI of KePLER).

R5: Reliability and fault-tolerance: Some computational environments are less reliable than others. For example, a workflow that incorporates a new web service can easily "break", as the latter can often fail, change its interface, or just become unacceptably slow (as it becomes more popular). To make a workflow more resilient in an inherently unreliable environment, contingency actions must be specifiable, e.g., fail-over strategies with alternate web services.

R6: User-interaction: Many scientific workflows require user decisions and interactions at various steps. ${ }^{7}$ For example, an improved version of PIW (Section 3.1.1) allows the user to inspect intermediate results and select and rerank them before feeding them to subsequent steps. An interesting challenge is the need for user interaction in a detached execution. Using a notification mechanism the user might

\footnotetext{
${ }^{7}$ In fact, when workflow management was still called "office automation", humans were the main processors of tasks - the workflow system was just used for book-keeping; cf. Section 3.3.
}

be asked to reconnect to the running instance and make a decision before the paused (sub)workflow can resume.

R7: "Smart" re-runs: A special kind of user interaction is the change of a parameter of a workflow or actor. For example, in a visualization pipeline or a long running workflow, the user might decide to change some parameters after inspecting intermediate or even final results. A "smart" re-run would not execute the workflow from scratch, but only those parts that are affected by the parameter change. In datafloworiented systems (e.g., visualization pipeline systems such as AVS, OpenDX, SCIRun, or the KEPLER system) this is easier to realize than in more control-oriented systems (e.g., business workflow systems), since data and actor dependencies are already explicit in the system. Another useful technique in this context is checkpointing, which allows to backtrack (in the case of a parameter change or even a system failure; cf. (R5)) to a previously saved state without starting over from scratch.

R8: "Smart" (semantic) links: A scientific workflow system should assist workflow design and data binding phases by suggesting which actor components might possibly fit together (this is also an aspect of (R2), service composition), or by indicating which data sets might be fed to which actors or workflows. To do so, some of the semantics of data and actors has to be captured. However, capturing data semantics is a hard problem in many scientific disciplines: e.g., measurement contexts, experimental protocols, and assumptions made are often not adequately represented. Even if corresponding metadata is available, it is often not clear how to best make it useable by the system. It seems clear though that ontologies provide a very useful semantic type system for scientific workflows, in addition to the current (structural) type systems [26].

R9: Data provenance: Just as the results of a conventional wet lab experiment should be reproducible, computational experiments and runs of scientific workflows should be reproducible and indicate which specific data products and tools have been used to create a derived data product. Beyond the conventional capture of metadata, a scientific workflow system should be able to automatically log the sequence of applied steps, parameter settings and (persistent 
identifiers of) intermediate data products. A related desiderata is automatic report generation: The system should allow the user to generate reports with all relevant provenance and runtime information, e.g., in XML format for archival and exchange purposes and in HTML (generated from the former, e.g., via an XSLT script) for human consumption.

Data provenance can be seen as a prerequisite to (R8): In order to provide semantic information about a derived data product, suitable provenance information is needed.

While the above list of requirements and desiderata for scientific workflow systems is by no means complete, it should be sufficient to capture many of the core characteristics. Other requirements include the use of an intuitive GUI to allow the user to compose a workflow visually from smaller components, or to "drill-down" into subworkflows, to animate workflow execution, to inspect intermediate results, etc.

A scientific workflow system should also support the combination of different workflow granularities. For example, coarse-grained workflows, akin to Unix pipelines or web service-based workflows, consist mainly of "black box" actors whose contents are unknown to the system. Scientific workflows may also be very fine-grained, or include fine-grained subworkflows. In that case, components are "white boxes" containing, e.g., the visual programming equivalent of an algorithm, or a system of differential equations to be solved, in other words, a detailed specification known to the system.

\subsection{Differences to Business Workflows}

The characteristics and requirements of scientific workflows are partially overlapping those of business workflows. Indeed, the term 'scientific workflows' seems to indicate a very close relationship with the latter, while a more detailed comparison reveals a number of significant differences. Historically, business workflows have roots going back to office automation systems of the 1970's and 80's, and gained momentum in the 90's under different names including business process modeling and business process engineering; see, e.g., $[12,77,83]$.

Today we see some influence of business workflow standards in the web services arena, specifically standards for web service choreography. ${ }^{8}$ For example, the

\footnotetext{
${ }^{8}$ Despite the long history of business workflows, it is surprising how short-lived some of the so-called standards are, as "most of them die before becoming mature" [75].
}

Business Process Execution Language for Web Services (BPEL4WS) [30], a merger of two earlier standards, IBM's WSFL and Microsoft's XLANG, has received some attention recently.

When analyzing the underlying design principles and execution models of business workflow approaches, a focus on control-flow patterns and events becomes apparent, whereas dataflow is often a secondary issue. For example, [76] describe a large number of workflow design patterns that can be used to analyze and compare business workflow standards and products in terms of their control features and expressiveness.

Scientific workflow systems, on the other hand, tend to have execution models that are much more dataflow-oriented. This is true, e.g., for academic systems including KePler, TAVERna [2], and TrIANA [3], and for commercial systems such as Inforsense's DiscoveryNet or Scitegic's Pipeline-Pilot. With respect to their modeling paradigm and execution models, these systems seem closer to an "AVS for scientific data and services" than to the more control-flow and task-oriented business workflow systems, or to their early scientific workflow predecessors $[28,54,11]$.

The difference between dataflow-orientation and control-flow orientation can also be observed in the underlying formalisms. For example, visualizations of business workflows often resemble flowcharts, state transition diagrams, or UML activity diagrams, all of which emphasize events and control-flow over dataflow. Formal analysis of workflows usually involves studying their control-flow patterns [41], and is often conducted using Petri nets.

Conversely, the underlying execution model of current scientific workflow systems usually resembles or is even directly implemented as a dataflow process network [39, 44], having traditional application areas, e.g., in digital signal processing. Dataflow-oriented approaches are applicable at very different levels of granularity, from low-level CPU operations found in certain processor architectures, to high-level programming paradigms such as flow-based programming [55]. Scientific workflow systems and visualization pipeline systems can also be seen as dataflow-oriented problem solving environments [80] that scientists use to analyze and visualize their data. Last not least, there is also a close relationship between dataflow-oriented approaches and (pure) functional languages, including non-strict variants such as Haskell (cf. Section 5.1). 


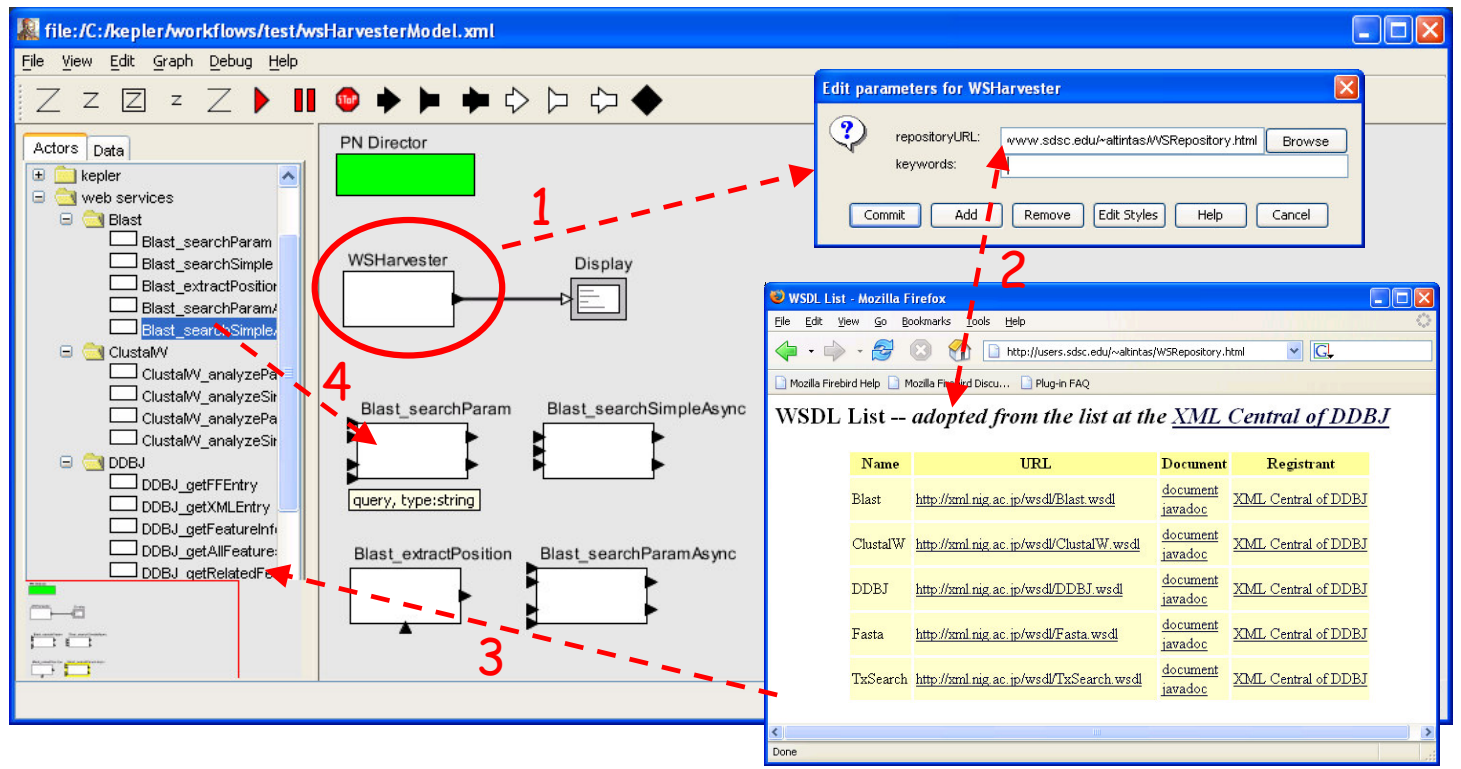

Figure 5: KePler web service HARVESTER in action: repository access (1-2), harvesting (3), and use (4).

\section{SPA Technology Development}

One of the recommendations coming out of the SciDAC/SDM review meeting in Napa Valley (March 2003) was to avoid working only as "consultants" to individual scientists, automating just their particular workflow needs. Instead, it was recommended to also focus on the development of generic technology (albeit guided by specific scientific applications). As a result of this, as well as the fact that the domain scientist $^{9}$ with whom we had worked intensively during the first report period (2001-2003) has subsequently been "adopted" by the SPA/LLNL team, we indeed focused our efforts on the development of such generic workflow technology.

In this section, we discuss some highlights of the current KEPLER system as well as some upcoming extensions. Many features directly address the requirements and desiderata from Section 3. More researchoriented extensions are described in Section 5.

\subsection{Web Service Extensions}

A basic requirement for scientific workflows is seamless access to remote resources and services (see (R1) in Section 3.2 and the examples in Section 3.1). Since web services are emerging as the standard means for remote service execution of loosely coupled systems, we extended KEPLER early on to handle web services. Given the URL of a web service description [81], the generic WebService actor of KePLER can be instantiated to any particular operation specified

\footnotetext{
${ }^{9}$ molecular biologist Matt Coleman, LLNL
}

in the service description. After instantiation, the WeBSERVICE actor can be incorporated into a scientific workflow as if it were a local component. In particular, the WSDL-defined inputs and outputs of the service are made explicit via the instantiated actor's input and output ports.

Figure 5 shows screenshots of an extended web service harvesting feature, implemented by a special web service HARVESTER component. ${ }^{10}$ As in the case of the generic WEBSERVICE actor, a URL is first provided (see (1) in Figure 5), however this time not to an individual WSDL description of a web service, but to a web service repository. The repository URL might point to a UDDI repository, or simply to a web page listing multiple WSDL URLs as shown in (2). The HARVESTER then retrieves and analyzes all WSDL files of the repository, creating instantiations of web service actors in the user's local actor library; see (3). For example, one of the harvested services, the BLAST web service, comprises five service operations which are imported into a corresponding subdirectory. The user can then drag-and-drop any of these service operations on the workflow canvas for use in a scientific workflow (4). The HARvester feature facilitates rapid prototyping and development of web service-based applications and workflows in a matter of minutes - that is, provided

(i) the web services are alive when needed, and

(ii) they can be wired together more or less directly to perform the desired complex task.

\footnotetext{
${ }^{10}$ Inspiration came from a similar feature in TAVERNA.
} 
The problem with (i) is that, while harvested web services look like local components, their runtime failure can easily "break" a scientific workflow, reminding the user that the service interface has been harvested, not the actual code. ${ }^{11}$ We are currently extending KEPLER to make workflows with web services more reliable. One simple approach is to avoid the association of a service operation with a fixed URL. Instead, a list of alternate services can be provided when the workflow is launched, and service failure can then be compensated by invocation of one of the alternate services. Another option is to insert special control tokens into the data stream, indicating to downstream actors the absence of certain results. Long running workflows may thus more gracefully react to web service failures and produce at least partial results. This idea has been further developed for "collection-oriented" (in the functional programming sense) workflows: via so-called "exception-catching actors", invalid (due to failures) data collections can be filtered out of the data stream, while valid subcollections pass through unaffected [53]. An interesting research question is how to extend Ptolemy II's pause-resume model to a full-fledge transaction model that can handle service failures.

The problem (ii) is even more fundamental and has different aspects: At the design level the challenge is how to devise actors that can be reused easily. In Section 4.3 we give a brief introduction to actor-oriented modeling, the underlying paradigm of PTolemy II, and discuss how it facilitates component composition and reuse. At the "plumbing" level it is often necessary to apply data transformations between two consecutive web services (called "shims" in TAVERna). Such data transformations are supported through various actors in KEPLER, e.g., XSLT and XQuery actors to apply transformations to XML data, or Perl and Python actors for text-based transformations.

\subsection{Grid and other Extensions}

Figure 6 depicts a number of KEPLER actors that facilitate scientific workflows, including workflows that make use of "the Grid". In the upper left, the previously discussed generic WEBSERVICE actor and some instantiations are shown. Note how the latter specialize their actor interface via their input/output ports: e.g., BlaSt_SEARChSimple has three input ports and one output port, for the search arguments and result, respectively. The naming scheme used is $W S N_{-} O P$, where $W S N$ is the name of the web service and $O P$ is a specific web service operation.

\footnotetext{
${ }^{11}$ Which is of course the whole point of web services.
}

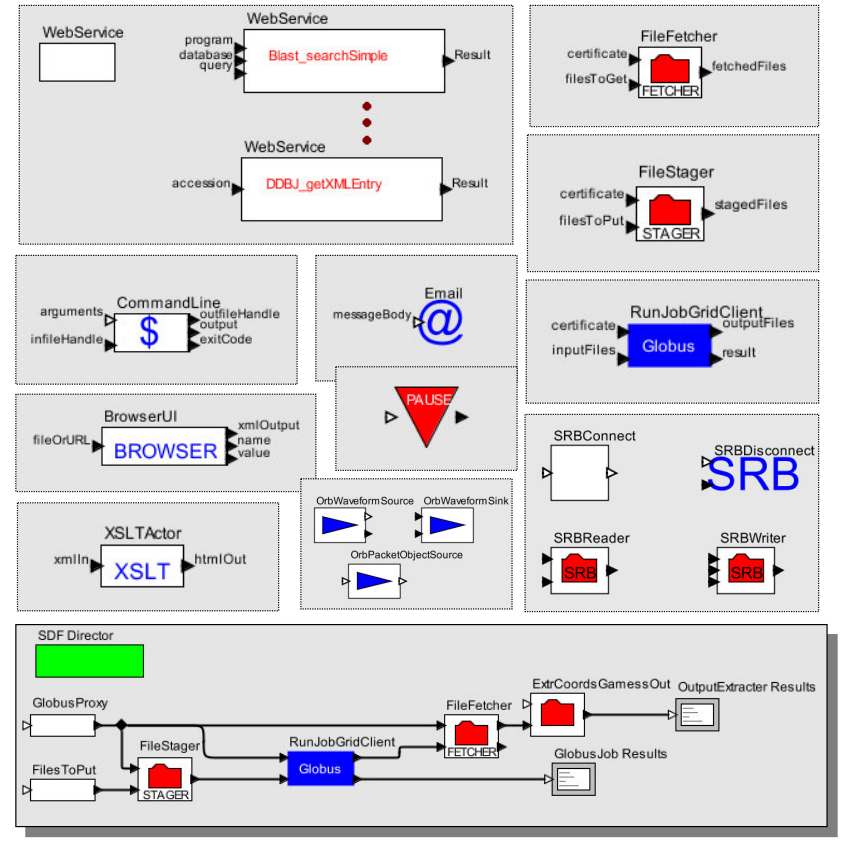

Figure 6: Grid actors and other KePLER extensions.

The upper right shows two Grid actors, called FileFetcher and FileStager, respectively. These actors make use of GridFTP [63] to retrieve files from, or put files to, remote locations on the Grid. The GlobusJob actor below is another Grid actor, in this case for running a Globus job [1]. At the bottom of Figure 6 a small workflow is shown that takes a Globus proxy and some input files, staging the files to where the job is run, then fetching the results from the remote location and displaying them on the client side. The green box specifies that this workflow is executed using an SDF (Synchronous Data-Flow) director. This director analyzes the dataflow dependencies and token consumption and production rates of actors (here: token $=$ file), and schedules the execution of actors accordingly.

On the right, a number of actors that use the SDSC Storage Resource Broker [72] are shown, e.g., to connect and disconnect from SRB and to get and put files from and to SRB space, respectively. We are currently in the process of providing all commonly used SRB commands as actors. This will allow the KEPLER user to design and execute Grid workflows involving a number of different tools, e.g., SRB for data handling aspects, and Globus, Nimrod and other tools for computational aspects and job scheduling.

In the center and left of Figure 6, various other KePler actors are shown: The CommandLine actor can be used to incorporate any application into a workflow, provided it can be accessed from the com- 
mand line. ${ }^{12}$ The "\$" icon is reminiscent of a shell prompt. The actor is parameterized with the arguments of the shell command, making it easy to create generic or specialized command line invocations. A Browser actor is shown directly below (cf. Section 3.1.2). It takes as input an HTML file or URL and displays it in the user's default browser. This makes the actor an ideal output device for displaying intermediate or final workflow results in ways that are well-known to users. Another extremely useful application of this actor is as an input device for user interactions. The result file of an upstream actor might have been transformed to an HTML file (e.g., using the XSLT actor) and augmented with HTML forms, check boxes, or other input forms that are displayable to the user in a standard web browser. Upon executing the desired user interaction, an http-post request is sent to a special KEPLER web server, acting as a listener, and from there the workflow is resumed.

The EMAIL actor in the center of the figure provides a simple notification mechanism to inform the user of specific situations in the workflow. Together, the EMAIL and BROWSER actors address core issues of requirement (R6) in Section 3.2. The PAUSE actor (red down-triangle) pauses workflow execution at specific points, allowing the user to inspect intermediate results, possibly changing parameter values, and resuming the workflow subsequently (addressing (R7) in Section 3.2).

Finally, actors for accessing real-time data streams from ROADNet sensor networks [67] have recently been added. These actors (e.g., OrBWAVEForMSoURCE) can be integrated easily into KEPLER, since many of the underlying PTOLEMY II directors support streaming execution. ${ }^{13}$

\subsection{Actor-Oriented Modeling}

Arguably the most unique feature of KEPLER comes from the underlying PTOLEMY II system:

\section{"The focus [of the Ptolemy project] is on as- sembly of concurrent components. The key underlying principle ... is the use of well- defined models of computation that gov- ern the interaction between components." 14}

This focus together with the actor-oriented modeling paradigm make PTOLEMY II an ideal starting point for tackling the breadth of challenges in scientific workflow design and execution. In Ptolemy, a system or

\footnotetext{
${ }^{12}$ E.g., KePLer workflows can include data analysis steps via calls to $\mathrm{R}[65]$.

${ }^{13}$ This should come as no surprise, since dataflow process networks are defined on token streams in the first place.

${ }^{14}$ http: //ptolemy.eecs. berkeley.edu/objectives.htm.
}

model thereof (in our case, a scientific workflow) is viewed as a composition of independent components called actors. Communication betweem actors happens through interfaces called ports. We distinguish between input ports and output ports. In addition to the ports, actors have parameters, which configure and customize the behavior. ${ }^{15}$ For example, a generic filter actor might consume a stream of input tokens via an input port, letting through to the output port only those tokens that satisfy a condition specified by a parameter.

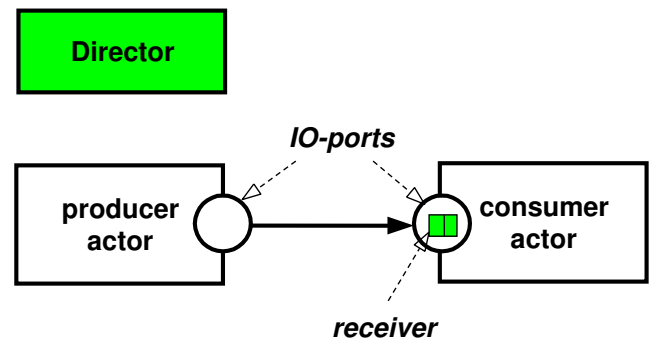

Figure 7: The semantics of component interaction is determined by a director, which controls execution and supplies the objects (called receivers) that implement communication.

Actors, or more precisely their ports, are connected to one another via channels. Given an interconnection of actors, however, there are many possible execution semantics that one could assign to the diagram. For example, actors might have their own thread of control, or their execution might be triggered by the availability of new inputs.

A key property of PTOLEMY II is that the execution semantics is specified in the diagram by an object called a director (see Figure 7). The director defines how actors are executed and how they communicate with one another. Consequently, the execution model is less an emergent side-effect of the various interconnected actors and their (possibly ad-hoc) orchestration, and more a prescribed concurrent semantics as one might find in a well-defined concurrent programming language. The execution model defined by the director is called the model of computation. Patterns of concurrent interaction are factored out into the design of the directors, rather than being individually constructed by the designer of the workflow. Figure 7 depicts a producer and a consumer actor whose ports are connected by a unidirectional channel. The diagram is annotated by a director, which might, for example, execute the producer prior to the consumer so as to respect data precedences. The communication between the actors is mediated by an object called a

\footnotetext{
${ }^{15}$ Parameters are usually not shown in the figures.
} 
receiver, which is provided by the director, not by the actors. Thus, for example, whether the communication is buffered or synchronous is determined by the designer of the director, not by the designer of the actor. This hugely improves the reusability of actor designs.

Process Networks. The Process Network (PN) director is a popular choice for designers of scientific workflows. It gives a diagram the semantics of (dataflow) process networks [39, 44]. In this semantics, actors are independent processes that execute concurrently, each with its own thread of control, and communicate by sending tokens through unidirectional channels with (in principle) unbounded buffering capacity. Writing to a channel is a nonblocking operation, while reading from a channel can block until sufficient input data are available. This model of computation is similar to that provided by Unix pipes, as in the following example of a Unix command-line composition of processes:

cat foo.txt | bar | baz

This example shows three independently executing processes (cat, bar, and baz) that are connected to one another through unidirectional pipes. The stream of tokens flowing between the processes also synchronizes them if necessary. For example if bar and baz are filter operations working on a single line of text at a time (e.g., grep $x y z$ ), then a Unix process executing bar will block until a line of text is provided by the process executing cat foo.txt. Unlike Unix pipes, however, the PN director in PTOLEMY IItolerates feedback loops and forking and merging of data streams It performs deadlock detection, and manages buffers to keep memory requirements bounded (if possible).

The PN director is only one example of a large number directors available in Ptolemy II. There is also, for example, the SDF (Synchronous Data-Flow) director, which can be used for specialized process networks with fixed token production and consumption rates per firing (see below). The SDF director performs static analysis on a workflow that guarantees absence of deadlocks, determines required buffer sizes, and optimizes the scheduling of actor execution. Other directors have been constructed for modeling Discrete Event systems (DE), Continuous-Time models (CT, which solve ordinary differential equations), and Communication Sequential Processes (CSP), to mention just a few [27].

By relieving actors from the details of component interaction, the actors themselves become much more reusable (cf. (R2) in Section 3.2). The behavior of an actor adapts to the execution and communication semantics provided by the director. This feature of actor-oriented modeling is called behavioral polymorphism. For example, a single Pтоlemy II actor implementation of an arithmetic operation, say PLus, can be connected to any number of input operands and reused within different models of computation and under the control of different directors. An SDF director, e.g., schedules the actor invocation (or "firing") as soon as all inputs have data, which it knows since actors declare their fixed token consumption and production rates in the SDF domain. In contrast, when the PLUS actor is governed by a DE director, additions happen when any input has data, corresponding to the different overall execution model in the Discrete Event domain. In addition to behavioral polymorphism, the P TOLEMY II type system also supports data polymorphism, again increasing the reusability of actors. For example, our PLUS actor can be implemented in such a way that it dynamically chooses the correct numeric addition (integer, float, double, complex), depending on the types of inputs it receives. Moreover, on other data types, e.g., strings, vectors, matrices, or user-defined types, the Plus actor ${ }^{16}$ can execute appropriate actions, e.g., string concatenation, vector or matrix addition, etc.

Actor-Oriented Programming Interface. Actororiented modeling addresses several challenges in the design of complex systems [34]. We have already mentioned improved component reusability due to behavioral and data polymorphism. Another aspect is hierarchical modeling. As illustrated by the examples in Section 3.1, subworkflows can be abstracted into (composite) actors themselves (e.g., see the CLASSIFIER actor/subworkflow in Figure 3) and thus arbitrarily nested. In the following, we give a simplified introduction on some implementation aspects of Ptolemy II's actor-oriented approach. These can be adapted to the context of scientific workflows and distributed, service-oriented environments, leading to a more structured approach to service composition and workflow design.

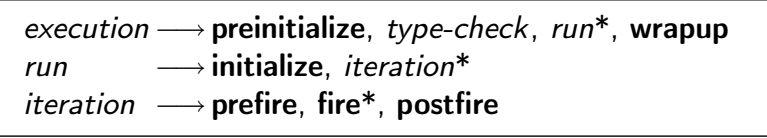

Figure 8: AOPI execution phases and actor methods.

The structure we propose is based on various phases and methods in PTOLEMY II's actor-oriented programming interface (AOPI), see Figure 8. These AOPI methods are used by a director to orchestrate overall

\footnotetext{
${ }^{16}$ This actor is called AddSubtract in Ptolemy iI.
} 
execution. Symbols in boldface denote actual methods that actor implementations have to provide; the remaining symbols describe other phases ${ }^{17}$ of the overall execution.

When a director starts a workflow execution, it invokes the preinitialize method of all actors. Since this method is invoked only once per lifetime of an execution (even if there are multiple runs), and prior to all other activities, this is a good time to put in place the receiver components of actors, and for actors to "advertise" their supported port data types, transport protocols, etc.

Next the director type-checks all connections and ports. This includes checking each port's data types, all (previously advertised) type constraints, and the validity of port types being connected through channels. A type inference algorithm is used to determine the most general types satisfying the given constraints. For scientific workflows, we can modify directors to also type-check which transport protocol to use, or to check whether producer and consumer actors exchange data directly or via handles: ${ }^{18}$ For example, if an actor A declares its output port to be of handle type "http | ftp" and a connected actor B declares its input port to be of handle type "http", then type-checking can establish that the connection is valid, provided A's output port is subtyped to use http handles only. Indeed such information can and should be passed to the actor with the invocation of the initialize method.

Other possible actions during execution of initialize are: Web service actors can "ping" the web services they represent and signal failure-to-initialize if the corresponding service is not alive. A "fail-overaware" director can use this information to replace the defective web service with an equivalent one that is alive (see (R5) in Section 3.2). A workflow execution will often consist only of one run, but if a workflow is re-run, initialize is called again. A run usually includes multiple iterations, each of which includes a call to prefire, fire (possibly called repeatedly by some special directors), and a call to postfire. The main actor operation finally happens in the fire method, e.g., a web service actor will make the actual remote service call here.

Towards Actor-Oriented Scientific Workflows. The idea of actor-oriented scientific workflows is to apply the principles of actor-orientation and hierarchical modeling, underlying the Ptolemy approach

\footnotetext{
${ }^{17}$ Some correspond to methods of other PTOLEMY II entities, e.g., director methods or manager methods [27].

${ }^{18}$ By handle we mean a unique identifier that can also be used to retrieve data, e.g., a URL.
}

$[34,27]$, to the modeling and design of scientific workflows. In particular, web service operations, which provide the building blocks of many loosely coupled workflows, should be structured into different parts, corresponding to the different phases and methods used in actor-oriented modeling. For example to implement a web service $\mathrm{w}_{\mathrm{A}}$, the service developer should think of specific web service operations such as $w_{A}$.initialize and $w_{A}$.prefire in addition to the main "worker" method $\mathrm{w}_{\mathrm{A}}$.fire. As in the case of Ptolemy actors, this will lead to more generic and reusable components and even facilitate more complex extensions such as stateful web services. ${ }^{19}$

\section{$5 \quad$ Research Issues}

In this section we briefly discuss some technical issues that we have begun addressing for KEPLER, but that are less mature and require some additional research.

\subsection{Higher-Order Constructs}

The early implementation of the Promoter Identification Workflow (PIW) depicted in Figure 2 demonstrated the feasibility and some advantages of implementing scientific workflows in the KEPLER extension of Ptolemy in [15]. However, it also highlighted some inherent challenges of the dataflow-oriented programming paradigm [48]. We have argued in Section 3.3 that many current scientific workflow systems are more dataflow-oriented than business workflow systems and approaches, which tend to emphasize event-based control-flow rather than dataflow. When designing real-world scientific workflows it is necessary, however, to handle complex control-flows within a dataflow-oriented setting as well. It is well-known that control-flow constructs require some thought in order to handle them properly. The fairly intricate network topology in Figure 2 includes backward-directed "dataflow" channels, having the sole purpose of sending control tokens that initiate another iteration of a subworkflow. While such complicated structures achieve the desired effect (here, a special kind of loop), they are hard to understand, design, and maintain. Such ad-hoc constructions also increase the complexity of workflow design while diminishing the overall reusability of workflow components (see (R2) in Section 3.2). Fortunately, there are better ways to incorporate structured control into a dataflow-oriented system, thereby directly supporting workflow design as required by (R2).

\footnotetext{
${ }^{19}$ Statefulness is an established concept in actor-oriented modeling and dataflow networks; e.g., it can be represented explicitly via feedback loops.
} 


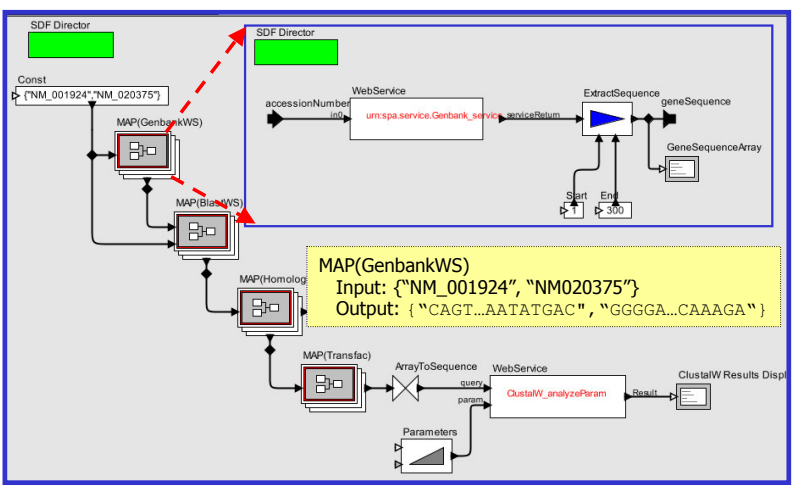

Figure 9: PIW variant with map iterator.

In [48] we have illustrated how higher-order functional programming constructs can be used to improve the design of PIW. In particular, the higherorder function map $::(\alpha \rightarrow \beta) \rightarrow[\alpha] \rightarrow[\beta]$ has proven to be very useful to implement a certain type of iteration. It takes a function $f$ (from $\alpha$ to $\beta$ ) and a list of elements of type $\alpha$, and applies $f$ to each list element, returning the list of result elements (each of type $\beta$ ). Thus map is defined as

$$
\operatorname{map} f\left[x_{1}, x_{2}, \ldots, x_{n}\right]=\left[f\left(x_{1}\right), f\left(x_{2}\right), \ldots, f\left(x_{n}\right)\right]
$$

For example, map $f[1,2,3]=[1,4,9]$ for $f(x)=x^{2}$.

Figure 9 shows an improved version of the PIW workflow from Section 3.1.1 and Figure 2, now using the higher-order map function. Note how backwarddirected flows of control-tokens are avoided. Instead, iterations are realized as nested subworkflows inside a higher-order MAP actor. For example, to implement a look-up of a list of gene sequences via a GenBank web service that can only accept one gene at a time, we simply create the higher-order construct MaP(GenBankWS) as shown in Figure 9 (the "stack" icon indicates that the contained workflow is applied multiple times).

Other higher-order functional programming constructs, e.g., foldr (for "fold right") can be similarly used to provide more abstract and modular iteration and control constructs in a dataflow setting, and we plan to add those to KEPLER in the future. The utility of declarative functional programming methods for dataflow-oriented systems is no coincidence; see, e.g., [66] for more on the close links between dataflow, functional, and visual programming, and [59] for interesting applications in implicit parallel programming. Here we only give a simple illustration using a core subworkflow of PIW in a Haskell specification; see [48] for details:

$$
\begin{aligned}
& \mathrm{d} 0=\$ \text { Gid } \\
& \mathrm{d} 1=\text { genBankG in } \\
& \mathrm{d} 2=\text { blastP } \mathrm{d} 1 \\
& \mathrm{~d} 3=\text { map genBankP d2 } \\
& \mathrm{d} 4=\text { map promoterRegion } \mathrm{d} 3 \\
& \mathrm{~d} 6=\text { zip } \mathrm{d} 2 \mathrm{~d} 4 \% \text { create list of (promoter-id, region) pairs } \\
& \mathrm{d} 7=\text { map gpr2str } \mathrm{d} 6 \quad \% \text { accumulate into string list } \\
& \mathrm{d} 8=\text { concat } \mathrm{d} 7 \\
& \mathrm{~d} 9=\text { putStr } \mathrm{d} 8 \\
& \% \text { create a single file }
\end{aligned}
$$

The input and output (ports) of this workflow are given by $d 0$ and $d 9$, respectively. Note the use of map to iterate over lists where the available services (e.g. genBankP) can only handle one item at a time. Also note that these ten equations establish a simple forward-only dataflow process network with the $\mathrm{d}_{i}$ representing named channels, and the expressions on the right of the equation representing processes (i.e., actors). A merge of two parallel branches happens, e.g., through the function zip that creates a single stream of pairs (promoter-id, promoter-region) in channel $\mathrm{d} 6$ from the two streams in $\mathrm{d} 2$ and $\mathrm{d} 4$.

\subsection{Third Party Transfers}

Scientific workflows can involve large volumes of data (see (R3) in Section 3.2). In a web service setting, this creates a problem since so-called 3rd party transfers are not currently supported by web services: Let us consider two web services $w_{A}$ and $w_{B}$, located at two sites $\mathrm{s}_{1}$ and $\mathrm{s}_{2}$, respectively. $\mathrm{w}_{\mathrm{A}}$ takes some input $x$ and produces some data $d$ that we would like to pass on to $w_{B}$, which produces the final output data $y$. We can depict this as follows:

$$
\stackrel{x}{\rightarrow} \mathrm{w}_{\mathrm{A}} @ \mathrm{~S}_{1} \stackrel{d}{\longrightarrow} \stackrel{\mathrm{W}_{\mathrm{B}} @ \mathrm{~S}_{2}}{\stackrel{y}{\rightarrow}}
$$

Assume that the overall execution of this workflow WF is coordinated and controlled by a workflow engine $\mathrm{E}$ (e.g., KePler) running at some site $s_{3}$. Current web service implementations do not allow the engine $\mathrm{E}$ to call $\mathrm{w}_{\mathrm{A}} @ \mathrm{~s}_{1}$, telling it to route $d$ directly to $\mathrm{w}_{\mathrm{B}} @ \mathrm{~s}_{2}$. Instead, web service invocations and the input/output dataflows that go with them, all go through $\mathrm{E}_{3}$. In pseudo-code this means:

$$
\begin{array}{r}
\text { WF@s } \mathrm{s}_{3}(\text { in } x, \text { out } y)=\{ \\
d @ \mathrm{~s}_{3}:=\mathrm{w}_{\mathrm{A}} @ \mathrm{~s}_{1}\left(x @ \mathrm{~s}_{3}\right) ; \\
\left.y @ \mathrm{~s}_{3}:=\mathrm{w}_{\mathrm{B}} @ \mathrm{~s}_{2}\left(d @ \mathrm{~s}_{3}\right)\right\}
\end{array}
$$

How do we execute the "remote assignments" shown

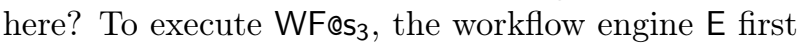
sends a request message containing $x$ to $\mathrm{w}_{\mathrm{A}} @ \mathrm{~s}_{1}$. Upon completion, $w_{\mathrm{A}}$ replies back to $\mathrm{E} \mathrm{s}_{3}$ with the result $d$. Now $\mathrm{WF} @ \mathrm{~s}_{3}$ can proceed and $\mathrm{E}$ forwards $d$ to $\mathrm{s}_{2}$ where $W_{B}$ can work on it. The final result $y$ is then sent 
from $s_{2}$ back to $s_{3}$. This simple call/return execution is quite desirable from a modeling and design point of view since control-flow and dataflow go hand in hand, and since the control engine $E$ does not have to worry about the status of direct (i.e., 3rd party) transfers of data $d$ from $\mathrm{w}_{\mathrm{A}}$ to $\mathrm{w}_{\mathrm{B}}$. The downside, however, is that data is moved around more often than necessary. Let us trace the "data shipments" of $x, d$, and $y$ :

1. ship $x @ \mathrm{~s}_{3} \sim x @ \mathrm{~s}_{1} \quad$ \% part of request to $\mathrm{w}_{\mathrm{A}}$

2. @ $\mathrm{s}_{1}$ execute $d:=\mathrm{w}_{\mathrm{A}}(x) \quad \%$ execute $\mathrm{w}_{\mathrm{A}}$

3. ship $d @ \mathrm{~s}_{1} \sim d @ \mathrm{~s}_{3} \quad$ \% part of reply from $\mathrm{w}_{\mathrm{A}}$

4. ship $d @ \mathrm{~s}_{3} \sim d @ \mathrm{~s}_{2} \quad$ \% part of request to $\mathrm{w}_{\mathrm{B}}$

5. @s ${ }_{2}$ execute $y:=\mathrm{w}_{\mathrm{B}}(d)$ $\%$ execute $\mathrm{w}_{\mathrm{B}}$

6. ship $y @ s_{2} \sim y @ s_{1}$ $\%$ part of reply from $\mathrm{w}_{\mathrm{B}}$

If $d$ is very large, executing both steps (3) and (4) is wasteful: first $d$ is sent from $\mathrm{s}_{1}$ to $\mathrm{s}_{3}$ where the workflow engine $E$ runs, only to be sent to $s_{2}$ in the next step. Instead of sending $d$ over the wire twice, the more direct 3rd party transfer $\mathrm{w}_{\mathrm{A}} @ \mathrm{~s}_{1} \stackrel{d}{\sim} \mathrm{w}_{\mathrm{B}} @ \mathrm{~s}_{2}$ moves $d$ only once, but as mentioned before, is not currently supported by web services. ${ }^{20}$ The question becomes: How can we avoid unnecessary transfers and achieve the efficiency of 3rd party transfer, while retaining the above simple call/return execution model?

A Handle-Oriented Approach. A simple solution to the above problem is that $\mathrm{w}_{\mathrm{A}}$ does not send the actual data $d$ but a handle $\mathrm{h}_{d}$ to it. Such a handle corresponds to a "logic pointer" and can be represented by a globally unique URI, but may also be a URL and indicate the protocol by which $d$ is to be accessed, e.g., http, ftp, GridFTP00, scp, or SRB. If we replace all data occurrences $x, d$, and $y$ by handles $\mathrm{h}_{x}, \mathrm{~h}_{d}$, and $\mathrm{h}_{y}$, respectively, we obtain:
1. ship $\mathrm{h}_{x} @ \mathrm{~s}_{3} \leadsto \mathrm{h}_{x} @ \mathrm{~s}_{1}$

2. @s $\mathrm{s}_{1}$ execute $\mathrm{h}_{d}:=\mathrm{w}_{\mathrm{A}}\left(\mathrm{h}_{x}\right)$

3. ship $\mathrm{h}_{d} \Subset \mathrm{s}_{1} \sim \mathrm{h}_{d} \Subset \mathrm{s}_{3}$

4. ship $\mathrm{h}_{d} @ \mathrm{~s}_{3} \sim \mathrm{h}_{d} @ \mathrm{~s}_{2}$

5. @s $s_{2}$ execute $h_{y}:=w_{B}\left(h_{d}\right)$

6. ship $\mathrm{h}_{y} @ \mathrm{~s}_{2} \sim \mathrm{h}_{y} @ \mathrm{~s}_{1}$
$\%$ request to $\mathrm{w}_{\mathrm{A}}$ $\%$ execute $\mathrm{w}_{\mathrm{A}}$ $\%$ reply from $\mathrm{w}_{\mathrm{A}}$ $\%$ request to $\mathrm{w}_{\mathrm{B}}$ $\%$ execute $\mathrm{w}_{\mathrm{B}}$

$\%$ reply from $\mathrm{w}_{\mathrm{B}}$

\footnotetext{
${ }^{20}$ And even if it were, "divorces" control-flow and dataflow, resulting in more complex execution models.
}

Now, instead of sending (the possibly very large) $d$ over the wire twice in (3) and (4), we only do so for the (constant size) handle $\mathrm{h}_{d}$. We cannot hope to further reduce this since a reply message from $\mathrm{w}_{\mathrm{A}}$ to $E$ and a new request from $E$ to $w_{B}$ are necessary for the overall control of workflow execution.

In order to implement the above handle-solution, we need to slightly extend our web services: in steps (2) and (5), $\mathrm{w}_{\mathrm{A}}$ and $\mathrm{w}_{\mathrm{B}}$ need to process handles by dereferencing them or by creating new ones. The former happens when a web service acts as a consumer of data ( $\mathrm{w}_{\mathrm{A}}$ consumes $x$ ), while the latter is needed in the role of a data producer ( $\mathrm{w}_{\mathrm{A}}$ produces $d$ ).

Consider, e.g., the case where handles are represented as URLs with http as the transport protocol. In step (2) above, $\mathrm{w}_{\mathrm{A}}$ needs to dereference $\mathrm{h}_{x}$ before it can execute its function. $\mathrm{h}_{x}$ might be, e.g., http://foobar.com/f17. When dereferenced via http-get it yields the actual data $x .^{21}$ To properly process handles as a data consumer, the operation "receive $x$ " has to be replaced by "receive $\mathrm{h}_{x}$ ", followed by a "dereference and get" operation $x:=\operatorname{http}-g e t\left(\mathrm{~h}_{x}\right)$. All subsequent read operations can then operate on $x$ as before.

In the role of a data producer, we have the reverse situation. We want to avoid shipping of the actual result data $d$ and instead send a handle $\mathrm{h}_{d}$. Thus, we need to first create this handle, e.g., by creating a new file $\mathrm{f} 18$ that can be accessed via $\mathrm{h}_{d}=$ http://baz.edu/f18. All subsequent write access to $d$ will proceed unchanged, provided the file name $\mathrm{f} 18$ is used for $d$. Finally, we need to replace "send $d$ " with "send $\mathrm{h}_{d}$ ".

We are currently working on extensions of KEPLER that make the system "handle-aware" [46]. For example, during the type-checking phase (Figure 8) a handle-aware director could determine whether two web service actors $A$ and $B$ that invoke the web services $w_{A}$ and $w_{B}$, respectively, support compatible handle types. For this to work seamlessly, web services themselves should offer an actor-oriented programming interface as presented in Section 4.3.

\subsection{Other Research Issues}

Higher-order constructs and the handle-approach to 3rd party transfers are only two of a number of pressing research issues in scientific workflows. ${ }^{22}$ For example, detached execution (R4), reliability and faulttolerance (R5), semantic links (R8), and data provenance (R9) are all scientific workflow requirements

\footnotetext{
${ }^{21}$ Note that while the handle $h_{x}$ is sent from $s_{3}$ to $s_{1}$ in step (1), $x$ might actually not reside at $s_{3}$.

${ }^{22}$ Addressing (R2) and (R3), respectively.
} 
that need further attention in the future. For example, [26] presents some initial work on the use of ontologies as semantic types to help generate data transformation mappings between consecutive workflow steps. These kinds of semantic extensions can help at both levels, at the "plumbing" level to create data transformations as in [26], and at the design level to create more reusable components (R2) and to support "smart" links in workflows (R8).

\subsection{Related Work}

In Section 4 we have described some of the features of KEPLER and the underlying PTOLEMY II system on which KePler is based. PtOlemy II aims at modeling and design of heterogeneous, concurrent systems. In contrast, KEPLER aims at the design and execution of scientific workflows. Consequently, KePler extensions to PтоLемY II include numerous actors and capabilities that facilitate scientific workflows (e.g., web service actors and harvester, GridFTP, SRB and database actors, command-line and secure shell actors, etc.) Additional components are constantly added th e.g., to support statistics packages (such as R), GIS functionality (e.g., Grass and ArcIMS couplings), and other scientific data analysis and visualization capabilities [78].

The research and development on KEPLER also benefits from interactions and collaborations with other groups. On one hand, development is driven by application scientists, the ultimate "customers" of scientific workflow system, on the other hand, work in related projects also influences KePLER developments. For example, TAVERna $[2,60]$ is a system that focuses on web service-based bioinformatics workflows. In contrast, Triana [3, 29] provides mechanisms for coupling workflows more tightly with Grid middleware tools. Cross-fertilization between these and other projects has happened, e.g., through e-Science LINKUP workshops [8], meetings and workshops at GGF [9], etc. Other scientific workflow tools include Pegasus [31], Chimera, and job scheduling tools such as Condor/G [33] and Nimrod/G [10]. For a taxonomy of workflow management systems for Grid computing and a comparison of systems see [82]. Future work will address the various outstanding research issues and workflows requirements that have not yet been (fully) met. For example, some projects contributing to KEPLER plan to provide couplings to highlyinteractive visualization tools such as SCIRun [78] and GeoVista [74].

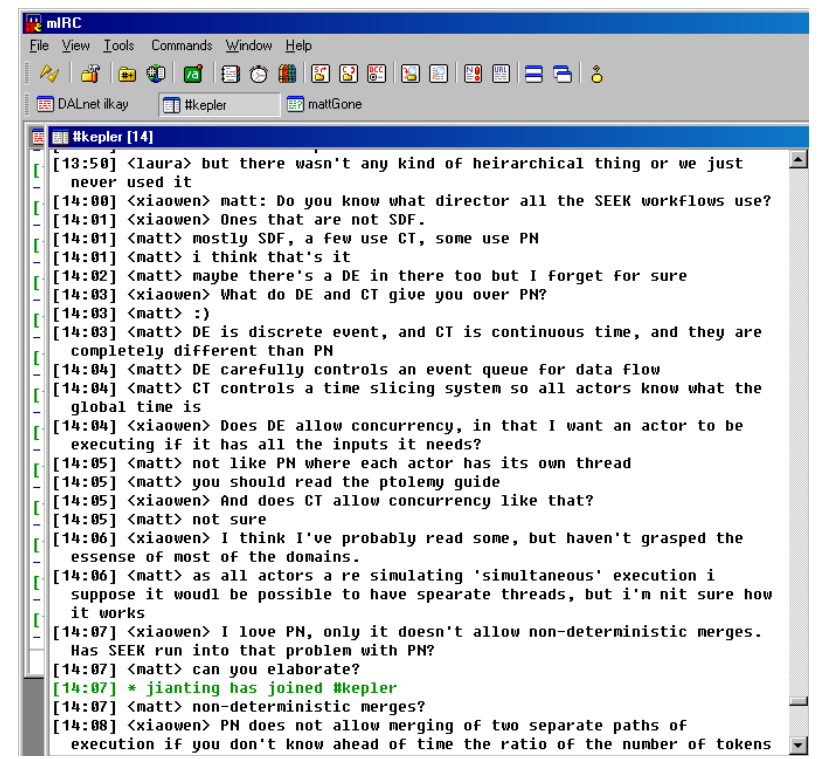

Figure 10: A screenshot of the KEPLER IRC channel. A member from Kepler/SPA (Xiaowen Xin) discussing with a Kepler/SEEK member (Matt Jones) , the technical issues surrounding the problem of a "non-deterministic merge".

\section{Brief History of the KEPLER Collaboration}

Towards the end of the first report period $(08 / 2001$ 08/2003) it became clear that significant progress towards a general scientific workflow tool would be difficult if not impossible by developing a system from scratch. Around the same time, the NSF/ITR project SEEK (Science Environment for Ecological Knowledge) had decided to base their development of a scientific workflow tool on the PTOLEMY II system [64]. During 2003, after several months of informal collaborations between developers from SEEK and from SciDAC/SDM, Dr. Ludäscher, a co-PI of both the SEEK and the SciDAC/SDM project, together with Matthew Jones, co-PI and project manager of SEEK, started discussions on how the collaboration could be organized to maximize leverage while preserving the different projects' individual needs. After initial discussions with Dr. Mladen Vouk from NCSU (whose students were, in addition to Ilkay Altintas from SDSC, the main SPA developers extending the Ptolemy II system for scientific workflows at the time), in November 2003 discussions between Arie Shoshani (SDM), Terence Critchlow (SDM), Bertram Ludäscher (SDM, SEEK), Mladen Vouk (SDM), and Matthew Jones (SEEK) led to founding of the open source KEPLER collaboration. 


\section{A Social Network Diagram for an IRC Channel}

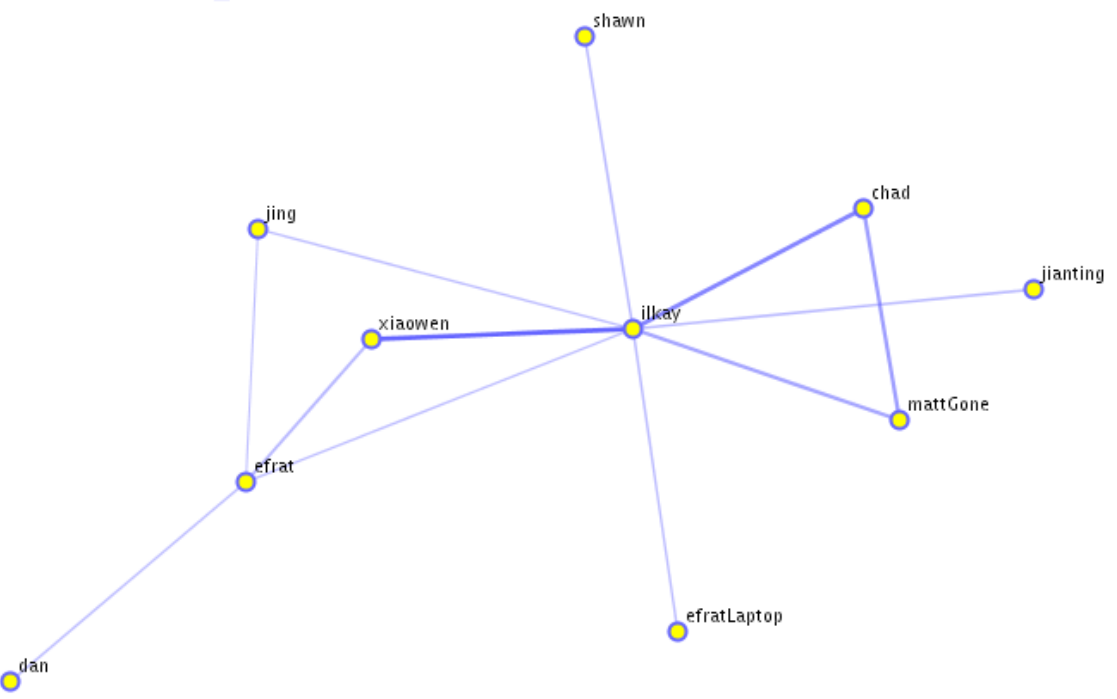

Figure 11: A social network obtained on Feb 15, 2005 from discussions on the KEPLER IRC channel. Here, Ilkay Altintas (Kepler/SPA, SDSC) has been a "hub" of a number of discussions with Xiaowen Xin (Kepler/SPA, LLNL), Shawn Bowers (Kepler/SEEK, UC Davis), and others.

KePLer Membership. Contributing members of the KEPLER collaboration are primarily developers but also researchers. There are different ways to become a member with read/write access to the shared CVS repository. For example, an existing member can "sponsor" a new member (thus being responsible for the new member's changes to the code). Once the new member has become familiar with the workings and practices of the existing team, the latter can vote the new member in as a regular member, thus removing the sponsorship status.

The Kepler Collaboration Today. Over time, a number of other projects teamed up with the original founding projects of KEPLER, i.e., SEEK and SPA: e.g., members supported under the NSF/ITR GEON project contributed workflows such as the one in Figure 3 and actors such as the SRB actors for large-scale data management (cf. Figure 6). Other examples include contributions from members of the NSF/ITR ROADNet project, the Resurgence cheminformatics project (SDSC and University of Zurich), the Encylopedia of Life project (SDSC), since recently also the NSF GeoStreams project (UC Davis), and last not least the original Ptolemy II project (UC Berkeley).

Today, KEPLER is a highly active collaboration, with regular developers meetings as well as meetings that interlink with other projects. For example, in addition to SDM All-Hands and PI Meetings, Supercomputing conferences, and other meetings, recent KEPLER and SPA specific meetings include:

- July 2004, SPA developers meeting (with several other KEPLER members attending), SDSC ${ }^{23}$

- October 2004, e-Science Link-Up meeting, SDSC; this collaboration (Dr. Ludäscher is a co-PI) is with members of the UK e-Science MyGrid project to share experiences gained in different scientific workflow projects (esp. KEPLER and Taverna) ${ }^{24}$

- January 2005, Kepler developer meeting in Juneau, Alaska $^{25}$

- February 2005, SPA software engineering meeting (with several other KEPLER members attending), UC Davis ${ }^{26}$

- May 2005, Ptolemy Miniconference, featuring KePler, UC Berkeley

\footnotetext{
23 http://kbis.sdsc.edu/events/SPA-07-04/

${ }^{24}$ http: //kbi.sdsc.edu/events/LINK-UP-10-04/

${ }^{25}$ http: //kepler-project.org/Wiki.jsp?page=

KeplerMeetingNotes January2004

${ }^{26}$ http: //kbi.sdsc . edu/events/SPA-Davis-02-05/
} 


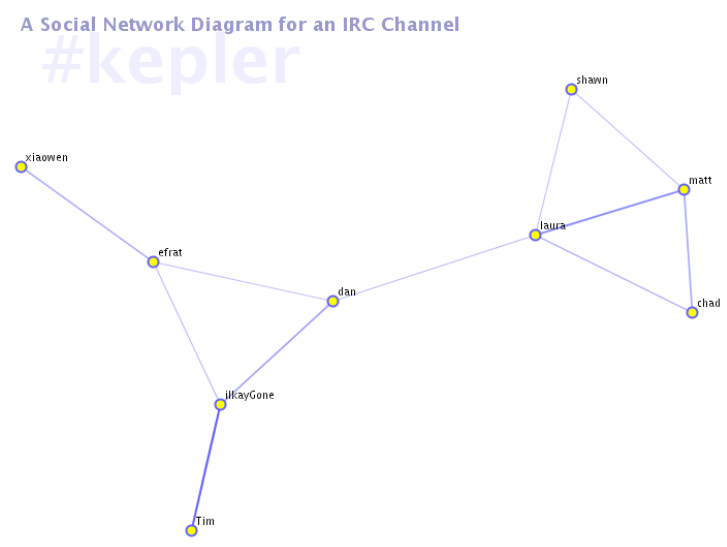

Figure 12: Social network obtained on March 7, 2005 from discussions on the KEPLER IRC channel. Ilkay Altintas (Kepler/SPA, SDSC), supposedly "gone" (but not quite) and Tim Wong (UC Davis, Bertram Ludäscher's ECS-289F student) form a very active link/discussion; similarly Laura Downey (Kepler/SEEK, UNM) and Matt Jones (Kepler/SEEK, UCSB). Also Xiaowen Xin (Kepler/SPA, LLNL) has been in contact with Efrat Frank (Kepler/GEON, SDSC).

- June 2005, Kepler developer meeting, Santa Barbara, (timed to to take advantage of SSDBM 2005)

Kepler Collaboration Tools. There are several ways by which KEPLER developers from various project regularly communicate to collaborate and co-develop the system. Standard mechanisms include of course email and a shared CVS repository (SPA also maintains a separate CVS repository for specific code that some SPA members might not want to share or contribute to KEPLER), but also voice-over-internet teleconferencing via Skype $^{27}$ and, in particular, internetrelay-chat (IRC). Figure 10 shows a screenshot of the KEPLER IRC channel, documenting a discussion between SPA and SEEK project members. The various interaction paths between team members are also depicted in Figures 11 and 12.

\section{Excerpts from the Quarterly Reports}

In this section we briefly highlight some of the detailed technical developments and accomplishments, roughly based on their chronological order as documented in the quarterly reports we have prepared for additional details please see the SDM quarterly reports themselves.

At the SDM/SPA meeting at Georgia Tech in May 2003, the decision had been made to abandon earlier efforts to start a SPA workflow system from scratch,

\footnotetext{
${ }^{27}$ http://www.skype.com
}

as it became clear that this would not be feasible within the time constraints and given the limited resources of the project. Moreover, a very suitable system was found in UC Berkeley's Ptolemy II system that had simultaneously been adopted as a starting point for a scientific workflow system by the SEEK NSF/ITR project. As indicated above, this choice has since proven an excellent one and first prototype workflows based on Ptolemy II could already been demonstrated at SSDBM'03 [15] and the SDM Framework workshop [5] and All-Hands Meeting, August 2003.

After adoption of Ptolemy II as the underlying system, technical development was often in the form of new or improved actors:

- New actors implemented: pause actor, interactive shell actor, initial version of web service actor.

- Initial work on an ant-based build system (in collaboration with Kepler/SEEK members).

Subsequently a number of actors were developed or refined:

- WSDL-based generic web service actor: Provides the user with an interface to connect and execute any web service defined by a WSDL URL.

- Initial version of Web service harvester: Imports all the operations of all the web services in a web service repository (e.g. UDDI) based on a keyword search. The operations execute as local actors when imported once. 
- XSLT transformation actor: allows transformations of XML and HTML generation from XML, using XSLT scripts.

- BrowserUI actor: a browser user interface can now be inserted anywhere in the workflow, allowing the user to display intermediate results (including text/HTML, SVG, etc. output), as well as submission of user interactions and selections through HTML forms.

- Database access and query actors: these allow access and manipulation of relational data.

- Grid ProxyInit, Globus Grid Job, and GridFTP actors: allow the user to create Grid jobs and to move large amounts of data round using Globus tools.

These were the main developments until the end of 2003; development continued in 2004 with the following items:

- New email actor: e.g., in long-running workflows, now an email can be sent to the user (including to email/SMS-enabled cell phones) about the status of the computation, including success, failure, or iteration count etc.

- New command line actor implementation started that allows users to invoke local commands and legacy tools.

- New SRB actors (provided by Efrat Jaeger Kepler/GEON); allow users access to SRB managed data collections.

- Updated Web Service actor: allow for primitive XML Schema types of WSDL-based generic web service actor (before: only support for xsd:string)

- Updated BrowserUI actor: The BrowserUI actor allows to launch any default browser on the client to provide workflow input and/or display workflow output (text/HTML, SVG, CGI, XML, etc.)

In addition, new workflows were developed and scientific communities were reached, in co-development with other KEPLER members:

- New Geologic Map Integration Workflow (implemented/operational); this workflow exhibits the use of web services and shows how a complex external system, here ESRI's ArcIMS is incorporated into the workflow (Altintas, Memon)
- New Mineral Classification Workflow (implemented/operational); this workflow shows how the workflow system can be used to re-engineer an existing end user scientist application. Includes loop constructs, SVG graphical output through the BrowserUI actor, and database access actor (Jaeger, Ludaescher)

- New Cheminformatics Workflow (designed using Kepler/SEEK workflow prototyping tool); once implemented this workflow will integrate Protein Databank (PDB) queries with the computational GAMESS biochemstry tool and the APBS tool; when implemented this will be an example of a "compute-intensive" workflow (Wibke Sudholt, I. Altintas)

The initial Kepler/SPA release was put together by Ilkay Altintas (SDSC) and Zhengang Cheng (NCSU). Later developments in 2004 include:

- New timestamp actor that outputs the system time.

- New SSH actor that implements the secure shell (ssh2) protocol and lets user natively connect to a server through secure shell.

- New generic JDBC-based DatabaseWriter and DatabaseReader actors that connect to any database and perform read, insert, update, delete and create table functions.

- New ExecutionLog utility: this is a separate workflow component that creates execution logs on the fly. This feature is designed to let the user post/save this log to a variety of grids/databases/files.

- Updated web service actor, web service harvester

- Created a WebStart installer for Kepler including the SPA modules.

- Designed and implemented a new documentation feature using taglets for describing actor signatures.

- New FileStager and FileFetcher actors, based on GridFTP.

- New Scp actor (secure copy). 


\section{Selected Publications}

We briefly highlight a few recent publications and presentations from the report period. See also the official SPA webpage ${ }^{28}$ for additional publications.

- [49] - overview paper on scientic workflow management and the KEPLER system; used also as a basis for this project report

- [16] - develops a framework for the design and reuse of "Grid workflows", i.e., having computeintensive and data-intensive aspects

- [47] - overview on scientific data management challenges, with a focus on data integration and semantics

- [19] - describes the computational chemistry prototyping environment and its use of KEPLER

- $[57,56,52,32]$ - work in database theory on the foundations of composing queries with limited access patterns; this is directly applicable to composing scientific workflows from web services

- [14] - presentation by Dr. Ludäscher at the Global Grid Forum Workshop on Scientific Workflows

- [26] - develops a new approach to employ semantic information in scientific workflows to guide structural data transformations

- $[17,42]$ - presentations on KePler at Supercomputing 2003 and 2004 by Ilkay Altintas and Werner Krebs, respectively

\section{Concluding Remarks}

We have provided an overview of scientific workflow management issues, an presented highlights of our work on award \#DE-FC02-01ER25486 on scientific workflow management during report period (20012007). This work is motivated by real-world needs and examples that we encountered in a number of application-oriented projects, in particular SDM/SPA. The spectrum of what can be called a scientific workflow is wide and includes scientific discovery workflows (e.g., Section 3.1.1), workflows that automate manual procedures or reengineer custom tools (e.g., Section 3.1.2), and data and compute-intensive workflows (e.g., Section 3.1.3). Scientific workflow support is needed for practically all information-oriented

\footnotetext{
${ }^{28}$ Formerly available at: http://www-casc.1lnl.gov/sdm/ see scholar.google.com/scholar?q=DE-FC02-01ER25486 for a current list.
}

scientific disciplines, including bioinformatics, cheminformatics, ecoinformatics, geoinformatics, physics, etc. We identified a number of common requirements and desiderata of scientific workflows (Section 3.2) and contrasted them with business workflows.

The KEPLER system addresses many of the core requirements (Section 4) and provides support for web service-based workflows and Grid extensions. The source code of KEPLER is freely available [40]; a first beta-release is in preparation (there have been several alpha-releases already). A unique feature of KEPLER is inherited from the underlying Ptolemy II system: the actor-oriented modeling approach. This approach facilitates modeling and design of complex systems and thus provides also a very promising direction for pressing problems such as web service composition and orchestration. The way data polymorphism and behavioral polymorphism are supported by an actororiented approach that "concentrates" component interaction in a separate director entity, can also shed light on other efforts to create reusable component architectures such as CCA [18]. Areas of research include modeling issues such as the use of higherorder functional constructs for workflow design (Section 5.1), and optimization issues such as the use of virtual data references (handles) to facilitate dataintensive, web service-based workflows (Section 5.2).

The research and development on KePLER does not occur in isolation. For example, cross-fertilization comes from interactions and collaborations with many groups; on one hand, this includes application scientists which are our ultimate "customers", on the other hand, this includes colleagues working on related projects. For example, TAVERna [2] is a system that focuses on web service-based bioinformatics workflows. In contrast, TRIANA provides mechanisms for coupling workflows more tightly with Grid middleware tools. Other scientific workflow tools include Pegasus, Chimera, and job scheduling tools such as Condor/G and Nimrod/G. Future work will address the various outstanding research issues and workflows requirements that have not yet been (fully) met. For example, some projects contributing to KEPLER plan to provide couplings to highly-interactive visualization tools such as SCIRun ${ }^{29}$ and to GIS systems such as GRASS ${ }^{30}$ and PostGIS ${ }^{31}$. A major challenge for the future will be a better integration between tightly coupled parallel applications and more loosely coupled scientific workflows.

\footnotetext{
${ }^{29}$ http://software.sci.utah.edu/scirun.html

${ }^{30}$ http://grass.itc.it/

${ }^{31}$ http://postgis.refractions.net/
} 


\section{References}

[1] The Globus Alliance. www.globus .org.

[2] The Taverna Project. http://taverna.sf .net/.

[3] The Triana Project. http://www.trianacode.org/.

[4] e-Science Workflow Services Workshop, e-Science Institute, Edinburgh, Scotland, December 2003. http: //www.nesc.ac.uk/esi/events/303/index.html.

[5] Scientific Data Management Framework Workshop, Argonne National Labs, August 2003. http://sdm. lbl.gov/ arie/sdm/SDM. Framework.wshp.htm.

[6] e-Science Grid Environments Workshop, e-Science Institute, Edinburgh, Scotland, May 2004. http: //www.nesc.ac.uk/esi/events/.

[7] GRIST Workshop on Service Composition for Data Exploration in the Virtual Observatory, California Institute of Technology, July 2004. http://grist.caltech.edu/sc4devo/.

[8] LINK-Up Workshop on Scientific Workflows, San Diego Supercomputer Center, October 2004. http: //kbis.sdsc.edu/events/link-up-11-04/.

[9] Workflow in Grid Systems Workshop, GGF10, Berlin, Germany, March 2004. http://www. extreme . indiana.edu/groc/Worflow-call.html.

[10] D. Abramson, J. Giddy, and L. Kotler. High Performance Parametric Modeling with Nimrod/G: Killer Application for the Global Grid. In Intl. Parallel and Distributed Processing Symposium (IPDPS), Cancun, Mexico, May 2000. http://www.csse.monash.edu.au/ davida/nimrod/.

[11] A. Ailamaki, Y. E. Ioannidis, and M. Livny. Scientific Workflow Management by Database Management. In 10th Intl. Conf. on Scientific and Statistical Database Management (SSDBM), Capri, Italy, 1998.

[12] G. Alonso and C. Mohan. Workflow Management Systems: The Next Generation of Distributed Processing Tools. In S. Jajodia and L. Kerschberg, editors, Advanced Transaction Models and Architectures. 1997.

[13] I. Altintas, O. Barney, and E. Jaeger-Frank. Provenance Collection Support in the Kepler Scientific Workflow System. Provenance and Annotation of Data (IPAW), pages 118-132, 2006. http://scholar . google. com/scholar?cluster $=10200865700598986854$.

[14] I. Altintas, C. Berkley, E. Jaeger, M. Jones, B. Ludäscher, and S. Mock. Kepler: Towards a GridEnabled System for Scientific Workflows. In Workshop on Workflow in Grid Systems, Global-Grid Forum (GGF10), Berlin, Germany, March 2004. .

[15] I. Altintas, S. Bhagwanani, D. Buttler, S. Chandra, Z. Cheng, M. Coleman, T. Critchlow, A. Gupta, W. Han, L. Liu, B. Ludäscher, C. Pu, R. Moore, A. Shoshani, and M. Vouk. A Modeling and Execution Environment for Distributed Scientific Workflows. In 15th Intl. Conf. on Scientific and Statistical Database Management (SSDBM), Boston, Massachussets, 2003.

[16] I. Altintas, A. Birnbaum, K. Baldridge, W. Sudholt, M. Miller, C. Amoreira, Y. Potier, and B. Ludäscher. A Framework for the Design and Reuse of Grid Workflows. In Intl. Workshop on Scientific Applications on Grid Computing (SAG'04), LNCS 3458. Springer, 2005. .

[17] I. Altintas and B. Ludäscher. A Modeling and Execution Environment for Scientific Workflows. Supercomputing 2003, Phoenix, AZ, November 2003. http://kbi.sdsc.edu/SciDAC-SDM/SC2003Slides . ppt.

[18] R. Armstrong, D. Gannon, A. Geist, K. Keahey, S. Kohn, L. McInnes, S. Parker, and B. Smolinski. Toward a Common Component Architecture for High-Performance Scientific Computing. In 8th IEEE Intl. Symposium on High Performance Distributed Computation, August 1999. 
[19] K. K. Baldridge, J. P. Greenberg, W. Sudholt, S. Mock, I. Altintas, C. Amoreira, Y. Potier, A. Birnbaum, K. Bhatia, and M. Taufer. The Computational Chemistry Prototyping Environment. In Proceedings of the IEEE, Special Issue on Grid Computing, 2005. in print.

[20] K. K. Baldridge, W. Sudholt, J. P. Greenberg, C. Amoreira, Y. Potier, I. Altintas, A. Birnbaum, D. Abramson, C. Enticott, and S. Garic. Cluster and Grid Infrastructure for Computational Chemistry and Biochemistry. In Parallel Computing for Bioinformatics, 2005. submitted for publication.

[21] Big Data is a Big Deal. Office of Science and Technology Policy, The White House, http://www . whitehouse.gov/blog/2012/03/29/big-data-big-deal, March 292012.

[22] Big Data is a Big Deal. Office of Science and Technology Policy, The White House, http://www. whitehouse.gov/sites/default/files/microsites/ostp/big_data_fact_sheet_final_1.pdf, March 292012.

[23] Biomedical Informatics Research Network Coordinating Center (BIRN-CC), University of California, San Diego. http://nbirn.net/.

[24] J. Blythe, E. Deelman, and Y. Gil. Planning for workflow construction and maintenance on the Grid. In ICAPS [38].

[25] S. Bowers, K. Lin, and B. Ludäscher. On Integrating Scientific Resources through Semantic Registration. In 16th Intl. Conf. on Scientific and Statistical Database Management (SSDBM), Santorini Island, Greece, 2004.

[26] S. Bowers and B. Ludäscher. An Ontology Driven Framework for Data Transformation in Scientific Workflows. In International Workshop on Data Integration in the Life Sciences (DILS), LNCS 2994, Leipzig, Germany, March 2004. .

[27] C. Brooks, E. A. Lee, X. Liu, S. Neuendorffer, Y. Zhao, and H. Zheng. Heterogeneous Concurrent Modeling and Design in Java (Volumes 1-3). Technical report, Dept. of EECS, University of California, Berkeley, 2004. Technical Memoranda UCB/ERL M04/27, M04/16, M04/17.

[28] I. Chen and V. Markowitz. The Object-Protocol Model: Design, Implementation, and Scientific Applications. ACM Transactions on Information Systems, 20(5), 1995.

[29] D. Churches, G. Gombas, A. Harrison, J. Maassen, C. Robinson, M. Shields, I. Taylor, and I. Wang. Programming Scientific and Distributed Workflow with Triana Services. Concurrency and Computation: Practice and Experience. Special Issue on Scientific Workflows, 2005.

[30] F. Curbera, Y. Goland, J. Klein, F. Leyman, D. Roller, S. Thatte, and S. Weerawarana. Business Process Execution Language for Web Services (BPEL4WS), Version 1.0, 2002. http://www.ibm.com/ developerworks/library/ws-bpel/.

[31] E. Deelman, J. Blythe, Y. Gil, C. Kesselman, G. Mehta, K. Vahi, K. Blackburn, A. Lazzarini, A. Arbree, R. Cavanaugh, and S. Koranda. Mapping Abstract Complex Workflows onto Grid Environments. Journal of Grid Computing, 1(1):25-39, 2003.

[32] A. Deutsch, B. Ludäscher, and A. Nash. Rewriting Queries using Views with Access Patterns under Integrity Constraints. In Intl. Conf. on Database Theory (ICDT), 2005. .

[33] T. T. Douglas Thain and M. Livny. Distributed Computing in Practice: The Condor Experience. Concurrency and Computation: Practice and Experience, 2004.

[34] J. Eker, J. W. Janneck, E. A. Lee, J. Liu, X. Liu, J. Ludvig, S. Neuendorffer, S. Sachs, and Y. Xiong. Taming Heterogeneity - the Ptolemy Approach. In Proceedings of the IEEE, volume 91(1), January 2003.

[35] J. Gao and M. A. Thompson, editors. Combined Quantum Mechanical and Molecular Mechanical Methods. American Chemical Society, 1998. 
[36] NSF/ITR: GEON: A Research Project to Create Cyberinfrastructure for the Geosciences, 2006. www. geongrid.org.

[37] A. Halevy. Answering Queries Using Views: A Survey. VLDB Journal, 10(4):270-294, 2001.

[38] Proceedings of the ICAPS Workshop on Planning for Web Services, Trento, Italy, June 2003.

[39] G. Kahn and D. B. MacQueen. Coroutines and Networks of Parallel Processes. In B. Gilchrist, editor, Proc. of the IFIP Congress 77, 1977.

[40] KePlen: A System for Scientific Workflows, 2006. http://kepler-project.org.

[41] B. Kiepuszewski. Expressiveness and Suitability of Languages for Control Flow Modelling in Workflows. $\mathrm{PhD}$ thesis, Queensland University of Technology, 2002.

[42] W. G. Krebs, I. Altintas, B. Ludäscher, P. Bourne, I. Shindyalov, and M. A. Miller. Integrated Genome Annotation Platform (iGAP): A Flexible, Modular System for Protein Sequence Annotation. In Supercomputing (SC), 2004.

[43] E. A. Lee and B. Ludäscher, editors. Sixth Biennial Ptolemy Miniconference - Featuring the Kepler Project, UC Berkeley, CA, May 2005. http://ptolemy.eecs.berkeley.edu/conferences/05/.

[44] E. A. Lee and T. Parks. Dataflow Process Networks. Proceedings of the IEEE, 83(5):773-799, May 1995. http://citeseer.nj.nec.com/455847.html.

[45] L. Liu, C. Pu, and W. Han. An XML-Enabled Data Extraction Tool for Web Sources. Intl. Journal of Information Systems, Special Issue on Data Extraction, Cleaning, and Reconciliation, 2001.

[46] B. Ludäscher. Towards Actor-Oriented Web Service-Based Scientific Workflows (or: How to Handle Handles). Technical report, San Diego Supercomputer Center, September 2004.

[47] B. Ludäscher. Managing Scientific Data: From Data Integration to Scientific Workflows. Göttinger Informatik Kolloquium, Georg-August-Universität, Göttingen, Germany, July 2005.

[48] B. Ludäscher and I. Altintas. On Providing Declarative Design and Programming Constructs for Scientific Workflows based on Process Networks. Technical Report SciDAC-SPA-TN-2003-01, San Diego Supercomputer Center, 2003. http://kbi.sdsc.edu/SciDAC-SDM/scidac-tn-map-constructs.pdf.

[49] B. Ludäscher, I. Altintas, C. Berkley, D. Higgins, E. Jaeger, M. Jones, E. A. Lee, J. Tao, and Y. Zhao. Scientific Workflow Management and the Kepler System. Concurrency and Computation: Practice \& Experience, 18(10):1039-1065, 2006. http://scholar.google.com/scholar?cluster= 12184448208352068070 .

[50] B. Ludäscher, I. Altintas, and A. Gupta. Compiling Abstract Scientific Workflows into Web Service Workflows. In 15th Intl. Conf. on Scientific and Statistical Database Management (SSDBM), Boston, Massachussets, 2003. http://kbis.sdsc.edu/SciDAC-SDM/ludaescher-compiling.pdf.

[51] B. Ludäscher, A. Gupta, and M. E. Martone. A Model-Based Mediator System for Scientific Data Management. In Z. Lacroix and T. Critchlow, editors, Bioinformatics: Managing Scientific Data. Morgan Kaufmann, 2003.

[52] B. Ludäscher and A. Nash. Web Service Composition Through Declarative Queries: The Case of Conjunctive Queries with Union and Negation. In 20th Intl. Conf. on Data Engineering (ICDE), 2004.

[53] T. M. McPhillips. Pipelined scientific workflows for inferring evolutionary relationships. Natural Diversity Discovery Project, 2005. manuscript.

[54] J. Meidanis, G. Vossen, and M. Weske. Using Workflow Management in DNA Sequencing. In Intl. Conf. on Cooperative Information Systems (CoopIS), 1996. 
[55] J. P. Morrison. Flow-Based Programming - A New Approach to Application Development. Van Nostrand Reinhold, 1994.

[56] A. Nash and B. Ludäscher. Processing First-Order Queries with Limited Access Patterns. In ACM Symposium on Principles of Database Systems (PODS), Paris, France, June 2004. .

[57] A. Nash and B. Ludäscher. Processing Unions of Conjunctive Queries with Negation under Limited Access Patterns. In 9th Intl. Conf. on Extending Database Technology (EDBT), LNCS 2992, pages 422-440, Heraklion, Crete, Greece, 2004.

[58] National Center for Biotechnology Information (NCBI). http://www.ncbi.nlm.nih.gov/, 2004.

[59] R. S. Nikhil and Arvind. Implicit Parallel Programming in pH. Morgan Kaufmann, 2001.

[60] T. Oinn, M. Addis, J. Ferris, D. Marvin, M. Senger, M. Greenwood, T. Carver, K. Glover, M. R. Pocock, A. Wipat, and P. Li. Taverna: A tool for the composition and enactment of bioinformatics workflows. Bioinformatics Journal, 20(17):3045-3054, 2004.

[61] OWL Web Ontology Language Reference, W3C Proposed Recommendation, December 2003. www.w3. org/TR/owl-ref/.

[62] L. Peterson, E. Yin, D. Nelson, I. Altintas, B. Ludäscher, T. Critchlow, A. J. Wyrobek, and M. A. Coleman. Mining the Frequency Distribution of Transcription Factor Binding Sites of Ionizing Radiation Responsive Genes. In New Horizons in Genomics, DOE/SC-0071, Santa Fe, New Mexico., March 30April 12003.

[63] G. Project. GridFTP - Universal Data Transfer for the Grid, 2000. see http://www.globus.org/ datagrid/gridftp.html.

[64] Ptolemy II project and system. Department of EECS, UC Berkeley, 2004. http://ptolemy.eecs. berkeley.edu/ptolemyII/.

[65] R - Statistical Data Analysis. http://www.r-project.org.

[66] H. J. Reekie. Realtime Signal Processing: Dataflow, Visual, and Functional Programming. PhD thesis, School of Electrical Engineering, University of Technology, Sydney, 1995.

[67] ROADNet: Real-time Observatories, Applications and Data management Network, 2006. roadnet. ucsd.edu.

[68] M. Schmidt, K. Baldridge, J. Boatz, S. Elbert, M. Gordon, J. Jensen, S. Koseki, N. Matsunaga, K. Nguyen, S. Su, T. Windus, M. Dupuis, and J. Montgomery. The General Atomic and Molecular Electronic Structure System. Journal of Computational Chemistry, 14:1347-1363, 1993. cf. http://www.msg. ameslab.gov/GAMESS/GAMESS.html .

[69] Scientific Data Management Center (SDM). http://sdm.lbl.gov/sdmcenter/, 2006.

[70] NSF/ITR: Enabling the Science Environment for Ecological Knowledge (SEEK), 2006. seek. ecoinformatics.org.

[71] A. Sheth. Changing Focus on Interoperability in Information Systems: From System, Syntax, Structure to Semantics. In M. Goodchild, M. Egenhofer, R. Fegeas, and C. Kottman, editors, Interoperating Geographic Information Systems, pages 5-30. Kluwer, 1998.

[72] SDSC Storage Resource Broker, 2006. http://www.sdsc.edu/srb/.

[73] W. Sudholt, K. Baldridge, D. Abramson, C. Enticott, and S. Garic. Parameter Scan of an Effective Group Difference Pseudopotential Using Grid Computing. New Generation Computing, 22:137-146, 2004. 
[74] M. Takatuska and M. Gahegan. GeoVISTA Studio: A codeless visual programming environment for geoscientific data analysis and visualization. Computers and Geosciences, 28(2):1131-1144, 2002.

[75] W. van der Aalst. Don't go with the flow: Web services composition standards exposed. IEEE Intelligent Systems. Web Services - Been there done that? Trends 83 Controversies, Jan/Feb 2003. http:// tmitwww.tm.tue.nl/research/patterns/download/ieeewebflow.pdf.

[76] W. van der Aalst, A. ter Hofstede, B. Kiepuszewski, and A. Barros. Workflow Patterns. Distributed and Parallel Databases, 14(3):5-51, July 2003.

[77] W. van der Aalst and K. van Hee. Workflow Management: Models, Methods, and Systems (Cooperative Information Systems). MIT Press, 2002.

[78] D. Weinstein, S. Parker, J. Simpson, K. Zimmerman, and G. Jones. Visualization in the SCIRun Problem-Solving Environment. In C. Hansen and C. Johnson, editors, Visualization Handbook, pages 615-632. Elsevier, 2005.

[79] T. Werner. Target gene identification from expression array data by promoter analysis. Biomolecular Engineering, 17:87-94, 2001.

[80] H. Wright, K. Brodlie, and M. Brown. The Dataflow Visualization Pipeline as a Problem Solving Environment. In M. Göbel, J. David, P. Slavik, and J. J. van Wijk, editors, Virtual Environments and Scientific Visualization, pages 267-276. Springer, 1996.

[81] Web Services Description Language (WSDL) Version 1.2. http://www.w3.org/TR/wsdl12, June 2003.

[82] J. Yu and R. Buyya. A Taxonomy of Workflow Management Systems for Grid Computing. Technical Report GRIDS-TR-2005-1, Grid Computing and Distributed Systems Laboratory, University of Melbourne, 2005. http://www.gridbus.org/reports/GridWorkflowTaxonomy.pdf.

[83] M. zur Muehlen. Workflow-based Process Controlling. Logos Verlag, Berlin, 2004. 